\title{
ARTICLE Plasmacytoid dendritic cell deficiency in neonates enhances allergic airway inflammation via reduced production of IFN- $\alpha$
}

Min Wu${ }^{1}$, Liuchuang Gao ${ }^{1}$, Miao He ${ }^{1}$, Hangyu Liu ${ }^{1}$, Han Jiang ${ }^{1}$, Ketai Shi ${ }^{1}$, Runshi Shang ${ }^{1}$, Bing Liu ${ }^{2}$, Shan Gao ${ }^{3}$, Hebin Chen ${ }^{4}$, Feili Gong ${ }^{1}$, Erwin W. Gelfand ${ }^{5}$, Yafei Huang ${ }^{6}$ and Junyan Han ${ }^{1}$

Allergic asthma, a chronic inflammatory airway disease associated with type 2 cytokines, often originates in early life. Immune responses at an early age exhibit a Th2 cell bias, but the precise mechanisms remain elusive. Plasmacytoid dendritic cells (pDCs), which play a regulatory role in allergic asthma, were shown to be deficient in neonatal mice. We report here that this pDC deficiency renders neonatal mice more susceptible to severe allergic airway inflammation than adult mice in an OVA-induced experimental asthma model. Adoptive transfer of pDCs or administration of IFN-a to neonatal mice prevented the development of allergic inflammation in wild type but not in IFNAR1 ${ }^{-1-}$ mice. Similarly, adult mice developed more severe allergic inflammation when pDCs were depleted. The protective effects of pDCs were mediated by the pDC-/IFN-a-mediated negative regulation of the secretion of epithelial cell-derived CCL20, GM-CSF, and IL-33, which in turn impaired the recruitment of CDC2 and ILC2 cells to the airway. In asthmatic patients, the percentage of pDCs and the level of IFN-a were lower in children than in adults. These results indicate that impairment of pDC-epithelial cell crosstalk in neonates is a susceptibility factor for the development of allergeninduced allergic airway inflammation.

Keywords: Neonate; Plasmacytoid Dendritic Cells; Allergic airway inflammation; IFN-a; Airway epithelial cells

Cellular \& Molecular Immunology (2020) 17:519-532; https://doi.org/10.1038/s41423-019-0333-y

\section{INTRODUCTION}

Asthma is an airway inflammatory syndrome resulting from a number of endotypes. A prevalent form is allergic asthma, which is defined as an unbalanced adaptive immune response to inhaled allergens. Recent studies suggest that crosstalk between the innate and adaptive immune systems is central to the initiation and development of allergic immune responses in the lung.,

Allergic asthma often begins in early life, at a time when immune responses differ substantially from responses in adults.,4 In neonates, adaptive immune responses are often biased toward Th2 cell activation, which may explain the high vulnerability of infants and children to Th2 cell-mediated allergic diseases. DCs are a bridge between innate and adaptive immunity because they govern $T$ cell differentiation. The activities of neonatal DCs are likely to play a central role in shaping Th2-skewed immune responses.

Plasmacytoid DCs (pDCs) are a specific subset of DCs, are major type I IFN producers, and play important roles in antivirus responses. ${ }^{5,6}$ pDCs have been shown to induce respiratory tolerance to harmless inhaled antigens, ${ }^{7}$ and increasing evidence supports the notion that $\mathrm{pDCs}$ negatively regulate allergic airway responses. The underlying mechanisms include the induction of regulatory $\mathrm{T}$ cells and central tolerance ${ }^{8-10}$, and suppression of
ILC2 cell function through the secretion of IFN- $a .^{11}$ In humans, neonatal pDCs have been reported to have dramatically impaired responses to TLR agonists and produce much less IFN- $a$ than adult pDCs. ${ }^{12-16}$ We previously found that neonatal mice have few pDCs in their lungs and that the frequency of $\mathrm{OX}_{40 \mathrm{~L}^{+}} \mathrm{pDCs}$ did not change after respiratory syncytial virus (RSV) infection. ${ }^{17}$ Cormier et al. reported that there is limited recruitment of pDCs and IFN-a production following RSV infection in neonatal mice. ${ }^{18}$ However, the means by which this deficiency contributes to the development of allergic airway responses in neonates remains to be determined.

In the present study, we confirmed that neonatal mice developed more severe allergic airway inflammation compared to that developed in adult mice. According to the results of adoptive pDC transfer into neonatal mice or depletion of pDCs in adult mice, pDCs were shown to be capable of suppressing allergic airway responses. In parallel, following IFN-a administration in the neonatal mice or using IFN-a receptor 1-deficient $\left(\right.$ IFNAR $1^{-1-}$ ) mice, we determined that pDCs mediated immunosuppressive activity through IFN-a release, which suppressed the secretion of CCL20, granulocyte-macrophage colony-stimulating factor (GM-CSF), and IL-33 from epithelial cells, leading to impaired recruitment of $\mathrm{CDC} 2$ and ILC2 cells. In patients with

\footnotetext{
${ }^{1}$ Department of Immunology, School of Basic Medicine, Tongji Medical College, Huazhong University of Science and Technology, Wuhan, China; ${ }^{2}$ Department of Respiratory Medicine, Zhongnan Hospital of Wuhan University, Wuhan, China; ${ }^{3}$ Department of Respiratory Diseases, Wuhan Central Hospital, Tongji Medical College, Huazhong University of

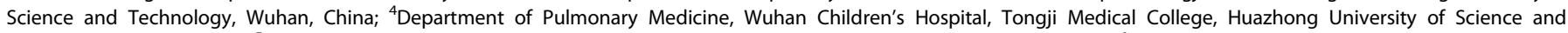

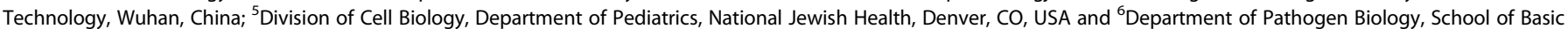
Medicine, Tongji Medical College, Huazhong University of Science and Technology, Wuhan, China

Correspondence: Junyan Han (hanj2014@hust.edu.cn)
}

Received: 22 March 2019 Revised: 7 November 2019 Accepted: 7 November 2019

Published online: 18 December 2019 
asthma, the pDC percentage in the sputum was lower in children than in adult patients, and this finding was associated with lower levels of IFN- $a$ in the children with asthma. These results indicate that the impairment of pDC-epithelial cell crosstalk in neonates is a susceptibility factor for the development of allergen-induced allergic airway inflammation.

\section{MATERIALS AND METHODS}

Mice

C57BL/6 mice were purchased from Beijing Vital River Laboratory Animal Technology Co., Ltd (Beijing, China). IFNAR1 ${ }^{-/-}$mice with a C57BL/6 background were kindly provided by Prof. Bo Zhong (University of Wuhan, Wuhan, China). The mice were housed under specific-pathogen-free conditions at Huazhong University of Science and Technology. The adult mice used in the experiments were female and 6-8 weeks of age. The neonatal mice were 7 days old. All experiments were approved by the animal ethics committee at Huazhong University of Science and Technology.

\section{Reagents}

Grade II and V ovalbumin (OVA) and aluminum hydroxide were purchased from Sigma (Sigma-Aldrich, St. Louis, MO). Fms-related tyrosine kinase 3 ligand (Flt3L) was purchased from Invitrogen (Thermo Fisher Scientific, Waltham, MA). The monoclonal 120G8 antibody was purchased from Lifespan Biosciences (Seattle, WA). Recombinant murine stem cell factor (SCF) was obtained from PeproTech (Rocky Hill, NJ). Recombinant mouse IFN-a was obtained from PBL Assay Science (Piscataway, NJ). IFN-a receptor blocking antibody (anti-IFN-aR1) was purchased from Santa Cruz Biotechnology (Weatherford, TX). IL-4, IL-5, IL-10, IL-17A, IFN- $\gamma$, GMCSF, and IgE ELISA kits were from BioLegend (San Diego, CA); IL-13 ELISA kit was from eBioscience (San Diego, CA); IL-33 and CCL20 ELISA kits were from R\&D System (Minneapolis, MN); and IgG1 and IgG2a ELISA kits were from Bethyl (Montgomery, TX). Mouse antibodies used for flow cytometry were anti-siglec-F (clone E502440; BD, San Jose, CA), anti-Ly6G (clone 1A8; BD), anti-CD11C (clone HL3; BD), anti-CD3e (clone 145-2C11; BD), anti-CD19 (clone 1D3; BD), anti-CD11b (clone M1/70; BD), anti-CD64 (clone X54-5/ 7.1; BD), anti-CD45 (clone 30-F11; BD), anti-CD90.2 (clone 53-2.1; $\mathrm{BD}$ ), anti-CD25 (clone PC61; BD), anti-ST2 (clone U29-93; BD), antiB220 (clone RA3-6B2; BD), anti-siglec-H (clone eBio440c; eBioscience), anti-IA/IE (clone 2G9; BD), anti-PDCA1 (clone eBio927; eBioscience), anti-CD103 (clone M290; BD), anti-CD4 (clone H129.19; BD), anti-FoxP3 (clone MF23; BD), anti-GARP (clone F011-5; BioLegend), anti-IL-10 (clone JES5-16E3; BD), anti-TGF- $\beta$ (clone TW7-20B9; BioLegend), anti-CD326 (clone G8.8; BD), and anti-CD31 (clone MEC 13.3; BD).

OVA-induced asthma model

Mice were sensitized on days 0 and 7 by two i.p. injections of OVA (grade V; Sigma) adsorbed to aluminum hydroxide (Sigma) (the dose was calculated according to body weight; $20 \mu \mathrm{g}$ OVA and 4 mg aluminum hydroxide in $100 \mu \mathrm{l}$ saline for $20 \mathrm{~g}$ body weight). ${ }^{19}$ Control mice were sensitized with saline. On days 15-19, the mice were challenged by inhalation of OVA ( $1 \% \mathrm{w} / \mathrm{v}$ in saline, grade II; Sigma) generated by a jet nebulizer for $30 \mathrm{~min}$. Twenty-four hours after the final challenge, the mice were euthanized for analysis.

As shown in Fig. 2, the neonatal mice were administered of PBS or Flt3L by i.p. injection ( $5 \mu \mathrm{g} / \mathrm{mouse}$ per day; Invitrogen) during sensitization. As shown in Fig. 4, the adult mice were administered (i.p. injection) IgG1 or monoclonal $120 \mathrm{G} 8 \mathrm{Ab}(100 \mu \mathrm{g} / \mathrm{mouse}$ per day; Lifespan Biosciences) during sensitization and before OVA challenge. As shown in Fig. 5, the neonatal mice were administered (i.p. injection) of PBS or recombinant mouse IFN-a $\left(5 \times 10^{3}\right.$ units/mouse per day; PBL Assay Science) twice before each sensitization and the first challenge.
Preparation of bronchoalveolar lavage fluid, lungs, and peribronchial lymph node cells

The mice were sacrificed and tracheostomized. The lungs were flushed with $1 \mathrm{ml}$ of PBS, and the bronchoalveolar lavage (BAL) fluid was collected and centrifuged at 1500 r.p.m. for $5 \mathrm{~min}$ at $4{ }^{\circ} \mathrm{C} .{ }^{20}$ The supernatants were collected for cytokine quantification by enzyme-linked immunosorbent assay (ELISA). The cells from the BAL fluid were resuspended in PBS and blocked with antiCD16/32 for $15 \mathrm{~min}$ and then stained with specific antibodies for FACS analysis (FACSVerse).

The lungs were perfused with PBS via the heart to clear the blood cells and then isolated and placed in cold PBS. These isolated lungs were cut into tiny pieces with scissors and digested with $1 \mathrm{ml}$ of digestion buffer (RPMl 1640 containing $5 \mathrm{mg} / \mathrm{ml}$ collagenase $\mathrm{D}$ [Roche, Carlsbad, CA] and $2 \mu \mathrm{M}$ EDTA [Ambion, Thermo Fisher Scientific, Waltham, MA]) for $30 \mathrm{~min}$ at $37^{\circ} \mathrm{C}$. Following digestion, $20 \mu \mathrm{l}$ of $0.5 \mathrm{mM}$ EDTA was added and maintained for $5 \mathrm{~min}$ at $37^{\circ} \mathrm{C}$. The lung cells were suspended and filtered through a $100-\mu \mathrm{m}$ cell strainer, and the red blood cells were lysed with lysing buffer. The lung cells were centrifuged at 1500 r.p.m. for $5 \mathrm{~min}$ at $4^{\circ} \mathrm{C}$, blocked with anti-CD16/32 for $15 \mathrm{~min}$, and then stained with specific antibodies for FACS analysis.

Peribronchial lymph node (PBLN) cells were isolated, ground, and pressed through a $70-\mu \mathrm{m}$ cell strainer to obtain single-cell suspensions. To determine cytokine production, the PBLN cells were cultured in complete RPMI 1640 medium at $1 \times 10^{6}$ cells $/ \mathrm{ml}$ and restimulated with OVA $(80 \mu \mathrm{g} / \mathrm{ml})$ for $72 \mathrm{~h}$. The supernatants were collected and stored at $-80^{\circ} \mathrm{C}$ for further examination.

\section{Flow cytometry and cell sorting}

Single-cell suspensions prepared from the BAL fluid and lungs were subjected to FACS staining. All cells were blocked with antiCD16/32 (clone 2.4G2; BD) and then stained with fluoresceinlabeled antibodies. The cells from the BAL were stained with antiCD11C, anti-siglec-F, anti-Ly6G, anti-CD3e, and anti-CD19. The lung DCs were stained with fluorescein-labeled antibodies against CD11c, CD11b, CD103, and MHC-II. The lung pDCs were stained with fluorescein-labeled antibodies against CD11c, CD11b, siglec$\mathrm{H}, \mathrm{B} 220$, and PDCA-1. Macrophages and granulocytes were stained with fluorescein-labeled antibodies against CD64, F4/80, CD11b, siglec-F, and Ly6G. Lymphocytes were stained with fluoresceinlabeled antibodies against CD45, CD3e, and CD19. Regulatory $T$ cells were stained with fluorescein-labeled antibodies against CD3e, CD4, CD25, FoxP3, and GARP. Flow cytometry was carried out with the FACSVerse (BD Biosciences). FlowJo software (Tree Star, Ashland, OR) was used to analyze the data.

To sort the epithelial cells, single-cell suspensions derived from the lungs were blocked with anti-CD16/32 and then stained with fluorescein-labeled antibodies against CD326, CD45, and CD31 and sorted on a FACS Aril III (BD Biosciences). ${ }^{21,22}$ The sorted cell populations were composed of $\mathrm{CD}^{-} 5^{-} / \mathrm{CD} 31^{-} / \mathrm{CD}_{2} 26^{+}$, but not leukocyte (CD45) or endothelial cells (CD31); however, lung epithelial cells $\left(\mathrm{CD} 326^{+}\right)$were captured. The purity of the sorted CD $45^{-} \mathrm{CD}^{-} 1^{-} \mathrm{CD} 326^{+}$cells was $\geq 90 \%$. The sorted epithelial cells were cocultured with OVA in the presence of IFN- $a$, the pDC culture supernatant, or the $\mathrm{pDC}$ culture supernatant with antiIFNAR1 mAb for $72 \mathrm{~h}$. The supernatants were collected, and the concentrations of IL-33, GM-CSF and CCL20 were determined by ELISA.

\section{Purification and adoptive transfer of pDCs}

Bone marrow cells from adult C57BL/6 mice were cultured in complete RPMI 1640 medium for 4 days with Flt3L $(200 \mathrm{ng} / \mathrm{ml}$; Invitrogen) and recombinant murine SCF $\left(50 \mathrm{ng} / \mathrm{ml}\right.$; PeproTech) ${ }^{23}$ at a concentration of $1 \times 10^{6}$ cells $/ \mathrm{ml}$. On day 5 , the nonadherent cells were collected and cultured in RPMI 1640 medium with 200 $\mathrm{ng} / \mathrm{ml} \mathrm{Flt3L}$. On day 10, the cells were collected, and the pDCs (defined as $\mathrm{CD} 11 \mathrm{c}^{+}$siglec- $\mathrm{H}^{+}$PDCA $-1^{+}$) were sorted by a FACSAria 
III flow cytometer (BD Biosciences). The purity of the sorted pDCs was $\geq 90 \%$ (as determined by flow cytometry). In the adoptive transfer experiment, $5 \times 10^{5}$ pDCs were injected (i.p.) into the neonatal mice one day before OVA sensitization.

\section{Lung histology}

The lung tissue was fixed in $4 \%$ paraformaldehyde and embedded in paraffin. The lung sections were stained with hematoxylin and eosin (H\&E) and periodic acid-Schiff (PAS). ${ }^{24}$ The samples were analyzed for inflammatory cell infiltration and goblet cell metaplasia.

Measurement of the cytokine and serum lg levels

The cytokine levels in the BAL fluid and the PBLN culture supernatants were measured using commercial ELISA kits according to the manufacturer's instructions.

Blood was collected and centrifuged at 7500 r.p.m. for $25 \mathrm{~min}$ at $4{ }^{\circ} \mathrm{C}$. The levels of total $\lg \mathrm{G} 1, \lg G 2 \mathrm{a}$, and $\lg \mathrm{E}$ in the serum were determined using ELISA kits.

\section{Lung homogenate collection}

The lungs were collected and homogenized with NP40 buffer. The homogenates were centrifuged at 10,000 r.p.m. for 10 min at $4{ }^{\circ} \mathrm{C}$. The concentrations of IL-33, CCL20. and GM-CSF in the supernatants were determined by ELISA.

\section{Human sputum samples}

Sputum samples used in this study were obtained from child and adult asthma patients from Wuhan Children's Hospital, Zhongnan Hospital of Wuhan University and the Central Hospital of Wuhan. The demographic characteristics of these patients are provided in Supplementary Tables I and II. All studies were approved by the ethics committee of Huazhong University of Science and Technology and were performed after written informed consent was obtained from all donors.

Sputum induction was performed as follows: (1) before induction, the mouth was washed with a gentle gargle with water, which was then expectorate the contents into a waste cup. (2) Subjects were given a 3.5\% saline via nebulizer, and the aerosol generation rate was adjusted to the tolerance of each subject to enable patient inhalation without discomfort. The subjects were asked to inhale the saline four times by mouth, and each saline inhalation was maintained for $2 \mathrm{~min}$. (3) Subjects were informed to not swallow sputum or saliva during the inhalation periods. At the end of each inhalation, the subject was instructed to expectorate saliva into the waste cup and to cough up sputum from the chest and expectorate it into the sputum cup. The subjects were closely observed throughout the inhalation periods to ensure that rescue therapy is given if needed.

The collected sputum samples were kept on ice during the entire induction procedure. Cellular mucus plugs were selected manually from sputum samples, weighed, and digested with a double volume of $0.1 \%$ dithiothreitol (DTT; Solarbio, Beijing; e.g., 1 $\mathrm{g}$ cellular mucus plugs in $2 \mathrm{ml}$ of DTT) at $37^{\circ} \mathrm{C}$ for $10 \mathrm{~min}$ to free sputum cells. Sputum cell suspensions were mixed gently and filtered with a $100-\mu \mathrm{m}$ cell strainer. Then, the cell suspensions were centrifuged at $4{ }^{\circ} \mathrm{C}$ and 1500 r.p.m. for $5 \mathrm{~min}$. The supernatants were collected and stored at $-80^{\circ} \mathrm{C}$, and the cells were suspended in staining buffer (PBS with 2\% FBS) for further investigation. The concentrations of human IFN- $a$ in the supernatants were determined by ELISA (DAKEWE, Beijing; detection range: $7.8-500 \mathrm{pg} / \mathrm{ml}$, sensitivity: $5 \mathrm{pg} / \mathrm{ml}$ ). Total counts of the cells in suspension were determined. A total of $2 \times 10^{6}$ sputum cells were blocked with an Fc receptor blocking solution (BioLegend, San Diego) for $15 \mathrm{~min}$ and then stained with fluorescently labeled CD11C, HLA-DR, CD123, and BDCA-4 antibodies for 30 min. Human pDCs were identified as CD $11 c^{\text {int }} \mathrm{HLA}-\mathrm{DR}{ }^{+} \mathrm{CD} 123^{+} \mathrm{BDCA}-4^{+}$. To decrease the contamination by basophils, we used HLA-DR expression to distinguish pDCs from basophils (because pDCs express high levels of HLA-DR, whereas basophils do not express HLA-DR). ${ }^{14,25}$ The frequencies of the different cells was determined by subjecting $200 \mu \mathrm{l}$ of $1 \times 10^{5}$ of suspended sputum cells to cytospin centrifugation using a modified Wright-Giemsa stain, as previously reported ${ }^{26} ; 400$ cells in several image frames were counted through a microscope by two individuals blinded to the details of the study. The total number of eosinophils and neutrophils in the sputum supernatant was calculated.

\section{Statistical analysis}

Experimental data were analyzed with GraphPad Prism (version 6.0, GraphPad Software, La Jolla, CA). Mann Whitney $U$ tests were used for comparing two groups using PRISM (GraphPad). Spearman correlation was used for the association analysis. The data are shown as the means \pm SD. $P<0.05$ was considered significant in all experiments.

\section{RESULTS}

Allergic airway inflammation and cellular responses are increased in neonatal mice

To test whether neonatal mice develop a more polarized Th2 cell response and have enhanced airway responsiveness compared to the adult mice, the neonatal and adult mice were sensitized with i.p. injections of OVA-aluminum hydroxide (OVA-alum) on day 0 and day 7 and then challenged with OVA via airway 1 week later (OVA/OVA; Fig. 1a). For the mice in both age groups, airway inflammation, as indicated by eosinophilia and infiltration of inflammatory cells around the airways and blood vessels, as well as goblet cell metaplasia, was induced following OVA sensitization and challenge (OVA/OVA); the OVA challenge (saline/OVA) alone was not effective (Fig. 1b, c). When the lung cell numbers were normalized to $100 \mathrm{mg}$ of lung weight, a direct comparison between the two age groups was possible (gating strategy see Supplementary Fig. 1), and the neonatal mice demonstrated greater eosinophilia (Fig. 1b) and goblet cell-rich lesions around the bronchus (Fig. 1c).

When PBLN cells were restimulated with OVA for 3 days, the Th2 cytokine levels were significantly higher in the OVA/OVA group compared to those of the saline/OVA control group. Higher levels of Th2 cytokines (IL-4, IL-5, and IL-13) and lower levels of the Th1 cytokine, IFN- $\gamma$, were produced by the PBLN cells from neonatal cells than were expressed by the adult PBLN cells (Fig. 1d).

We next examined serum Ig levels since IgE production is often associated with allergic airway inflammation and Th2 cell responses. Indeed, the OVA/OVA mice in both age groups demonstrated significantly higher total serum levels of IgE and IgG1 compared to the levels in the saline/OVA controls. When the two age groups were compared, the total serum levels of $\lg E$ and $\operatorname{lgG} 1$ were higher in the neonatal mice (Supplementary Fig. 2a-c). Collectively, these results indicate that, throughout airway inflammation, the production of Th2 cytokines by PBLN cells and $\lg E$ and $\lg G 1$ production can be induced in both mouse age groups upon OVA sensitization and challenge, these responses were enhanced to a greater extent in the neonatal mice than in the adults.

Lung DC and ILC2 cell characterization in neonatal vs adult mice As a bridge between innate and adaptive immunity, DCs are critical for initiating and polarizing $T$ cell responses. Lung DCs are heterogeneous and include $\mathrm{CD}_{11} \mathrm{~b}^{+}$conventional dendritic cells (cDCs), $\mathrm{CD}_{103^{+}} \mathrm{cDCs}$, and pDCs. We analyzed the different DC subsets in the lungs of the sensitized and challenged mice. The number of lung $C D 11 b^{+} c D C s$, an important antigenpresenting cell subset, was increased in the OVA-sensitized mice compared to the number in the saline-sensitized mice in both age groups. However, the number was lower in the neonatal group 

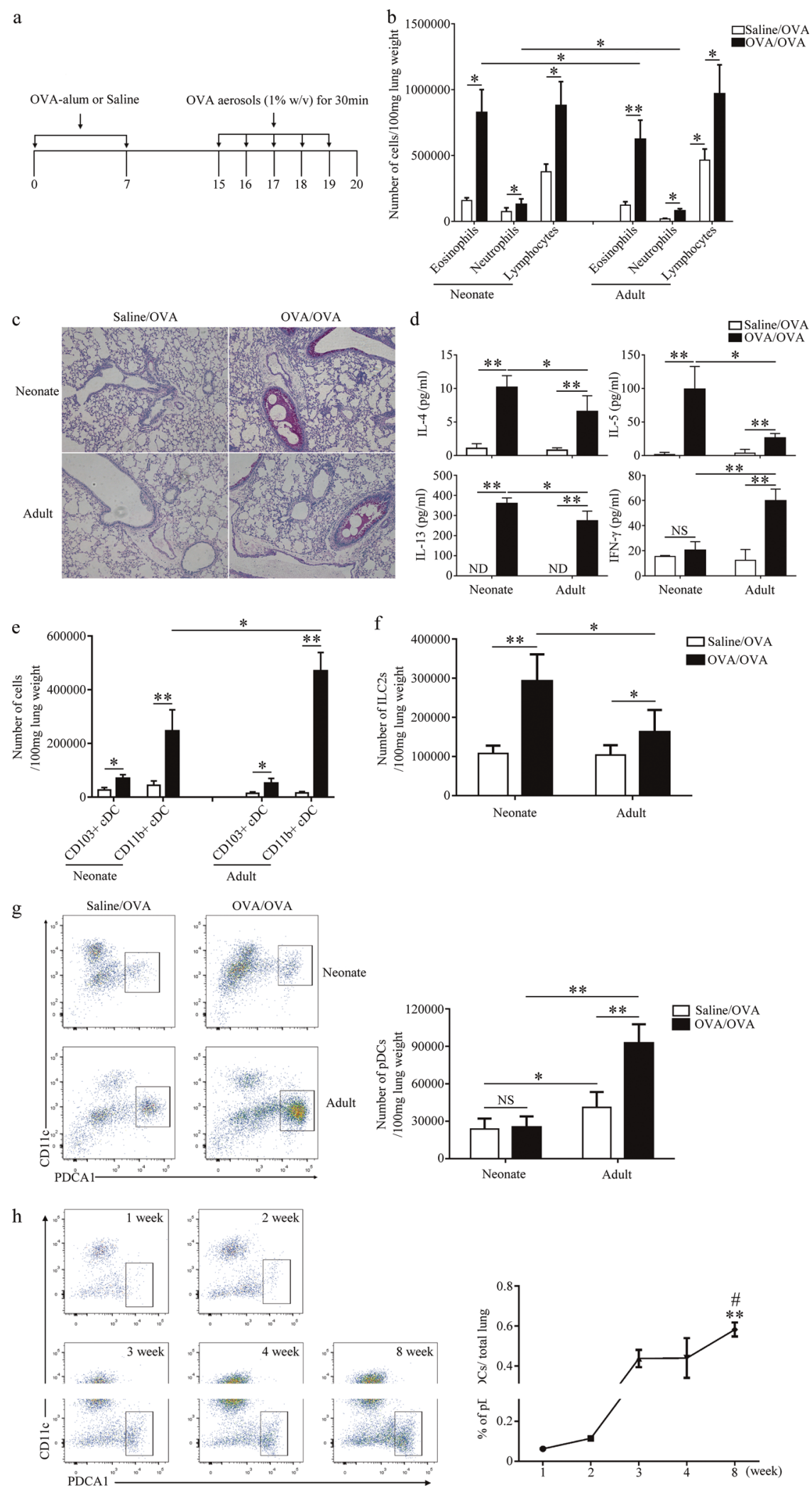

Fig. 1 Allergic airway inflammation was enhanced in neonatal mice. a Schematic of the allergic asthma model. Neonatal and adult mice were sensitized by two i.p. injections of OVA-alum on days 0 and 7 and then challenged with five OVA aerosol treatments on days 15-19. Mice were dissected, and the lungs were analyzed on day 20. b The number of eosinophils, neutrophils and lymphocytes in the lung was determined by flow cytometry. c PAS-stained lung sections. $\mathbf{d}$ Cytokine production in the supernatants of PBLN cells 3 days after restimulation with OVA in vitro. e-g The number of CDCs (e), ILC2 cells (f), and pDCs (g) in the lungs was determined by flow cytometry. $\mathbf{h}$ The percentage of pDCs in the lungs of mice from different age groups (**P<0.01, compared with 3-week-old mice; \#P<0.05, compared with 4-week-old mice). All data were analyzed with Mann Whitney $U$ tests without multiple comparison corrections. The results are presented as the means \pm SD ( $n=5-7$ each). ${ }^{*} P<0.05$. ${ }^{* *} P<0.01$. NS not significant, ND not detected. 


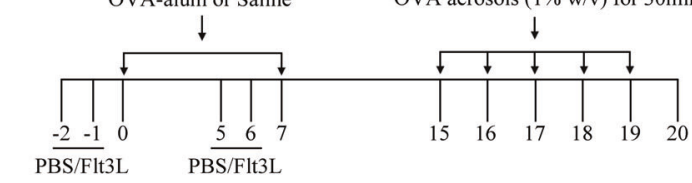

b
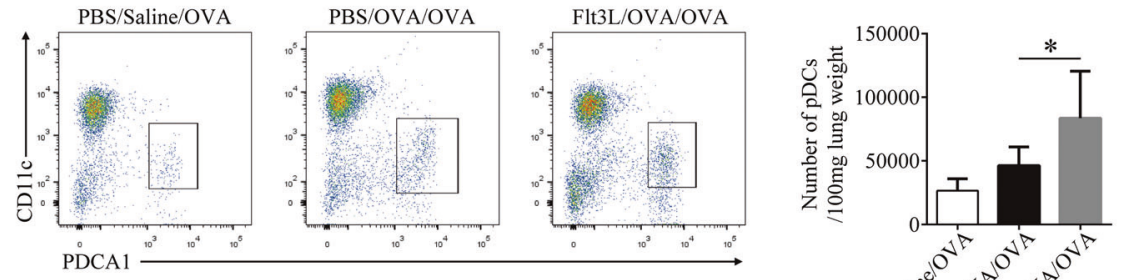

$\mathrm{c}$
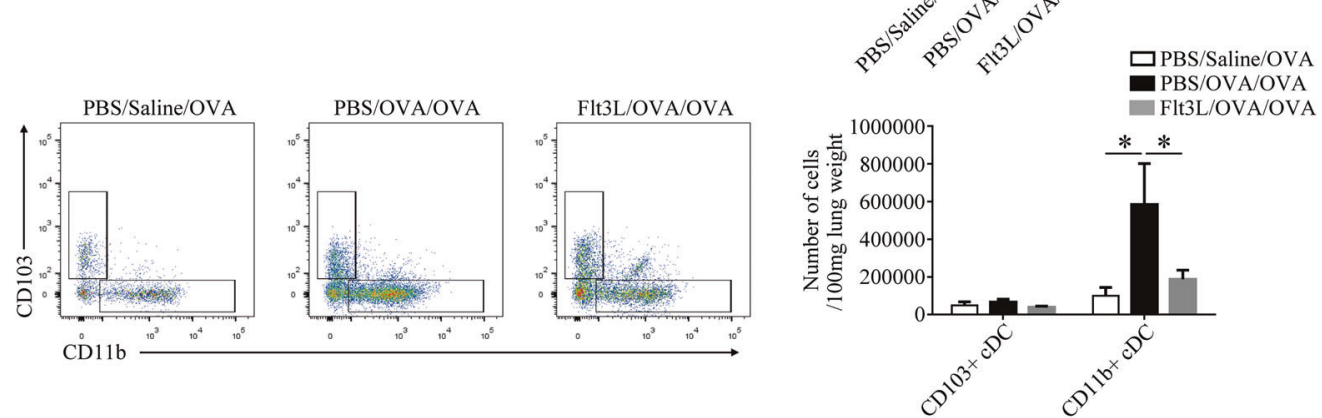

d

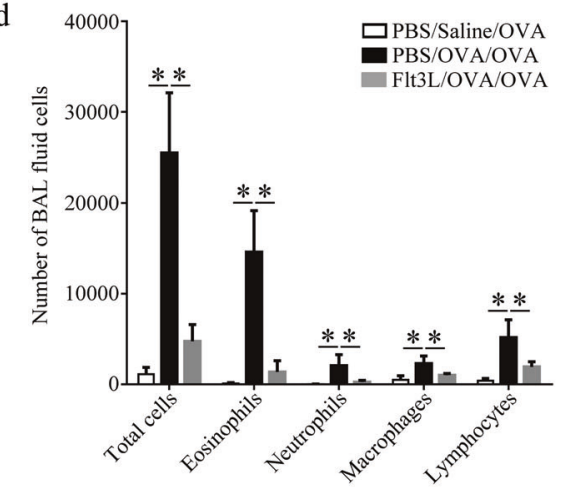

e $\quad$ PBS/Saline/OVA

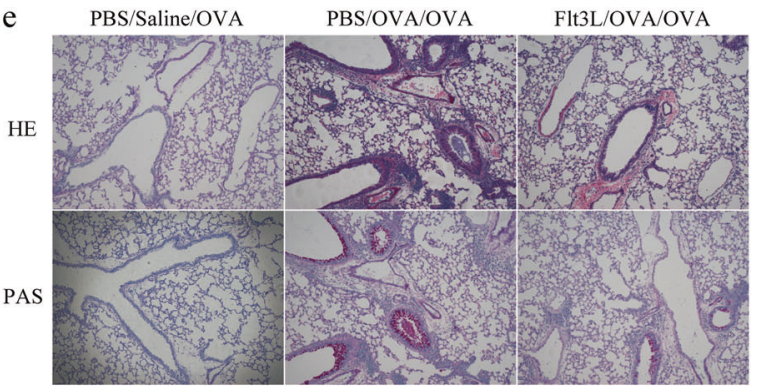

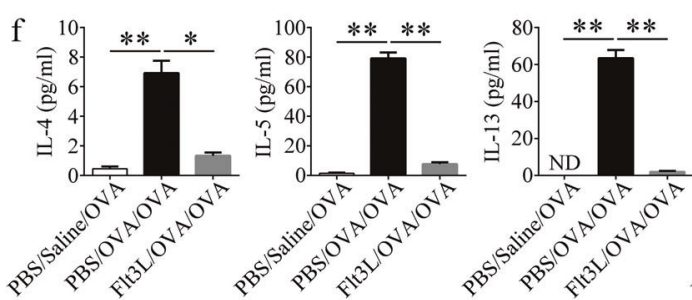
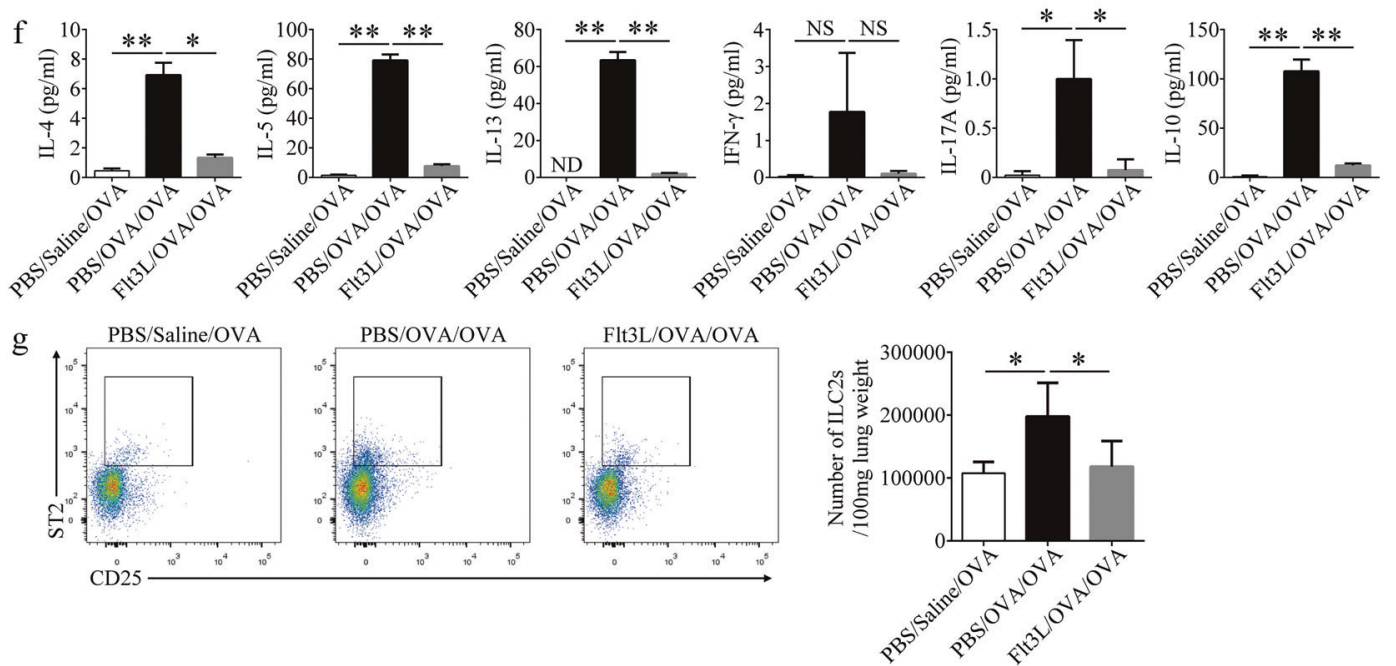

Fig. 2 Administration of Flt3L reduced the allergic airway responses in neonatal mice. a To replenish the pDCs, the neonatal mice were treated with Flt3L ( $5 \mu \mathrm{g}$ i.p. injection) twice before each OVA sensitization or with PBS as a control. Mice were dissected, and the lungs were analyzed on day 20 . b, $\mathbf{c}$ The number of $\mathrm{pDCs}(\mathbf{b})$ and $\mathrm{CDCs}(\mathbf{c})$ in the lung was determined by flow cytometry. $\mathbf{d}$ BAL cellularity was determined by flow cytometry. e HE- and PAS-stained lung sections. $\mathbf{f}$ Cytokine production in the supernatants of the PBLN cells 3 days after restimulation with OVA in vitro. $\mathbf{g}$ The number of ILC2 cells in the lung was determined by flow cytometry. All data were analyzed with Mann Whitney $U$ tests without multiple comparison correction. The results are shown as the means \pm SD $\left(n=6-8\right.$ each). ${ }^{*} P<0.05 .{ }^{* *} P<0.01$. NS not significant, ND not detected. 
$\mathrm{M}$ Wu et al.

a

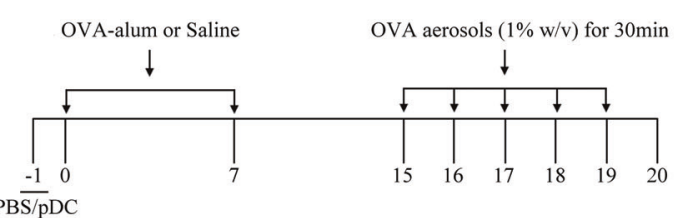

b
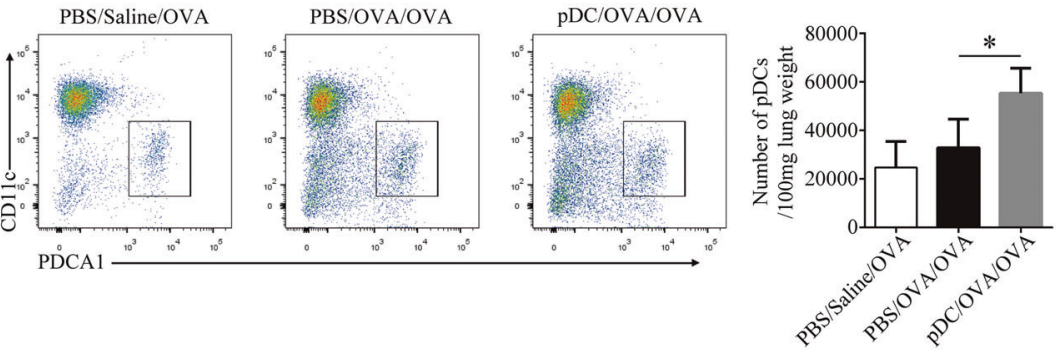

c
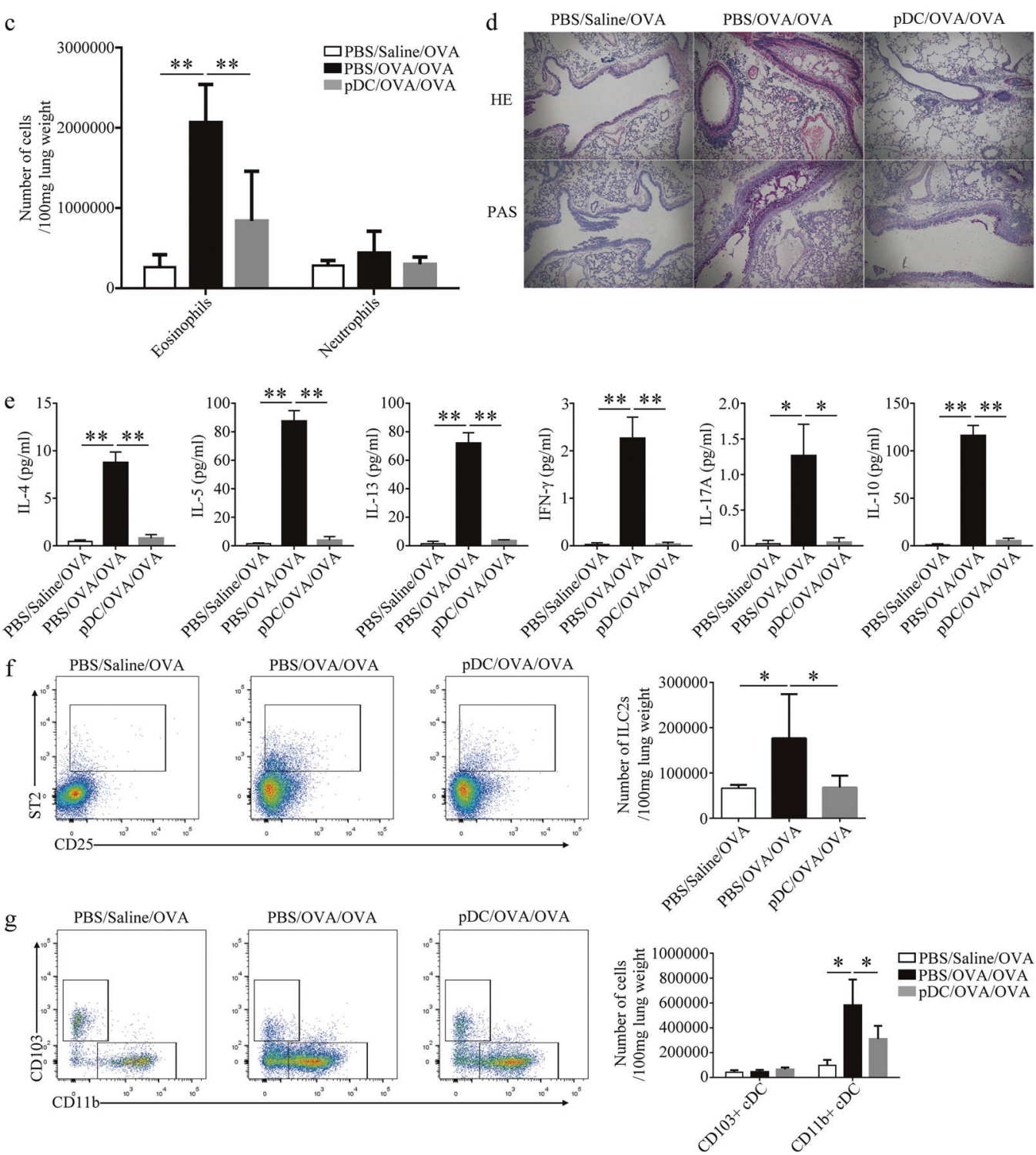

Fig. 3 Adoptive transfer of pDCs to neonatal mice reduced allergic airway responses. a One day before the first OVA sensitization, the neonatal mice received pDCs $\left(5 \times 10^{5}\right.$ /mouse) or PBS as a control. b The number of pDCs in the lung was determined by flow cytometry. c The number of eosinophils and neutrophils in the lung was determined by flow cytometry. $\mathbf{d}$ HE- and PAS- stained lung sections. e Cytokine production in the PBLN cell supernatants 3 days after restimulation with OVA in vitro. $\mathbf{f}, \mathbf{g}$ The number of ILC2 cells (f) and cDCs ( $\mathbf{g})$ in the lung was determined by flow cytometry. All data were analyzed with Mann Whitney $U$ tests without multiple comparison correction. The results are shown as the means \pm SD $\left(n=6-8\right.$ each). ${ }^{*} P<0.05$. ${ }^{* *} P<0.01$. 
a

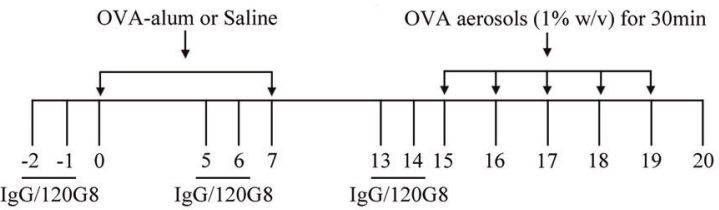

b
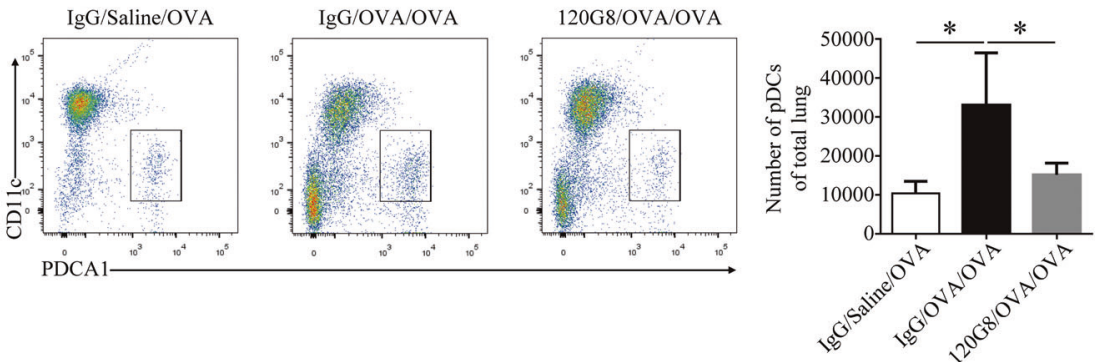

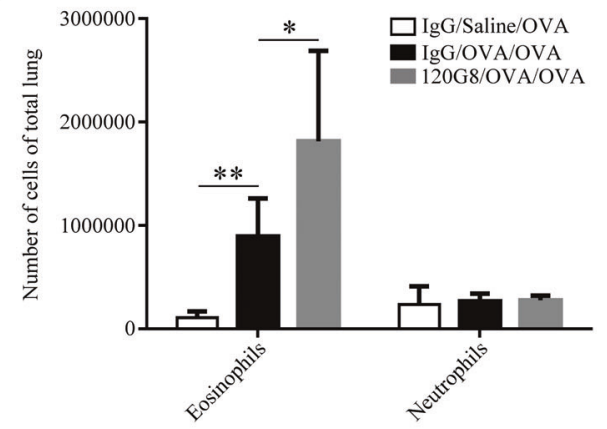

d IgG/Saline/OVA IgG/OVA/OVA

120G8/OVA/OVA

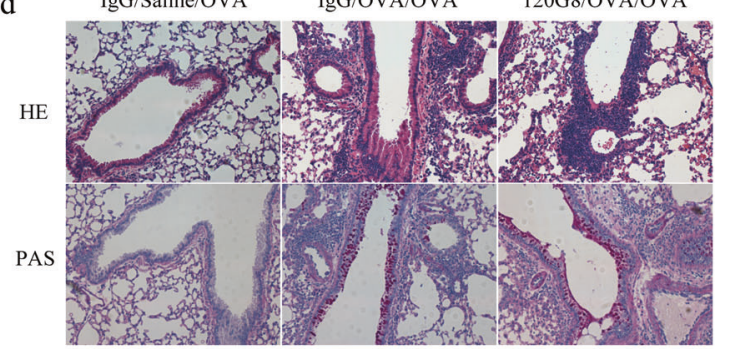

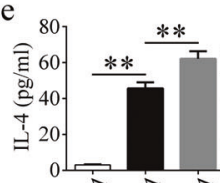
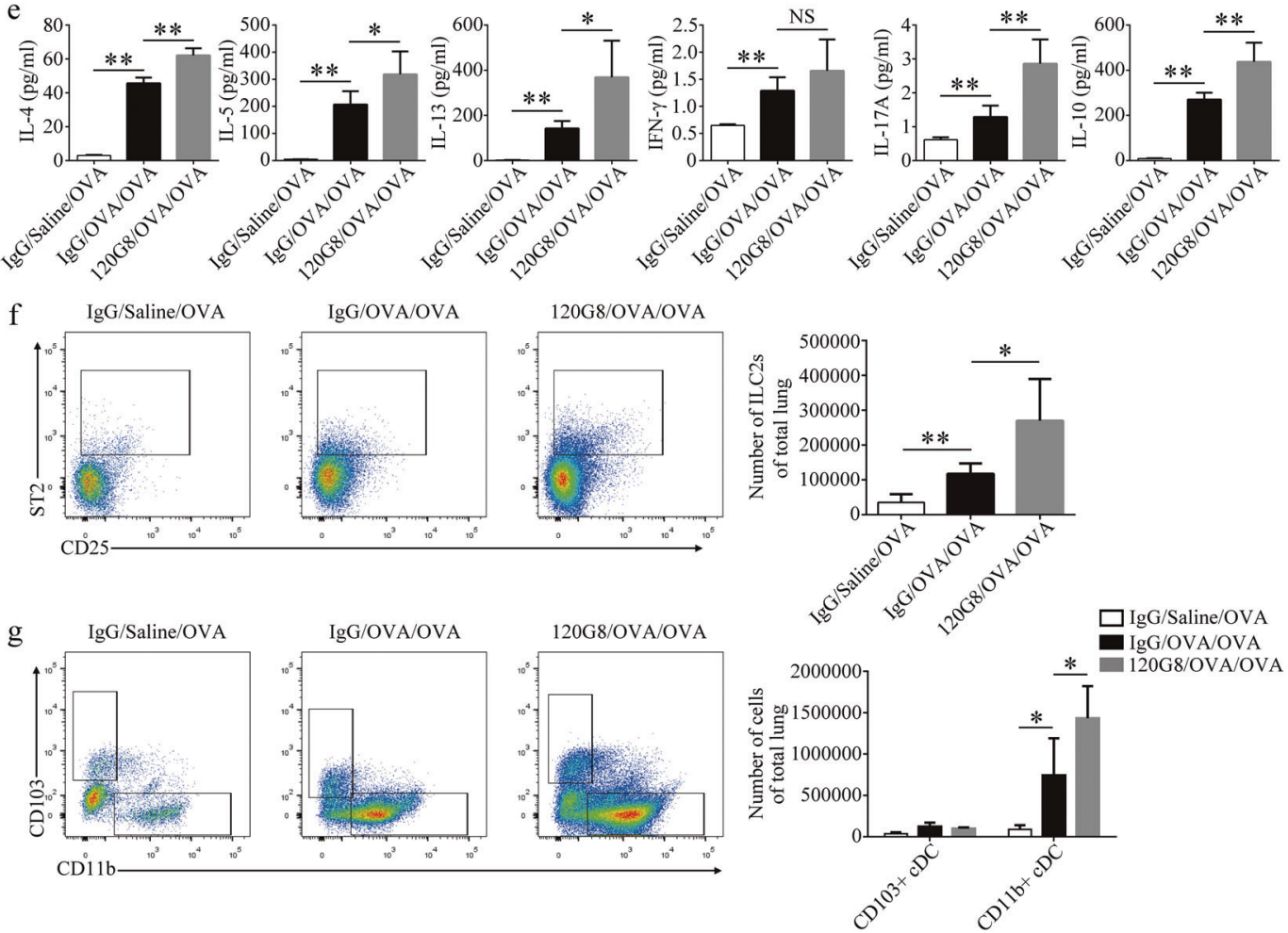

Fig. 4 Depletion of pDCs in adult mice exacerbated allergic airway responses. a To deplete the pDCs, the adult mice were treated with monoclonal Ab 120G8 (100 $\mu \mathrm{g})$ twice before each sensitization and the first challenge. The mice treated with lgG served as the control. $\mathbf{b}$ The number of pDCs in the lung was determined by flow cytometry. $\mathbf{c}$ The number of eosinophils and neutrophils in the lung was determined by flow cytometry. d HE- and PAS-stained lung sections. e Cytokine production in the supernatants of the PBLN cells 3 days after restimulation with OVA in vitro. $\mathbf{f}, \mathbf{g}$ The number of ILC2 cells (f) and CDCs $(\mathbf{g})$ in the lung was determined by flow cytometry. All data were analyzed with Mann Whitney $U$ tests without multiple comparison correction. The results are shown as the means \pm SD $\left(n=4-5\right.$ each). ${ }^{*} P<0.05 .{ }^{* *} P<0.01$. NS not significant. 
$\mathrm{M} \mathrm{Wu}$ et al.

a

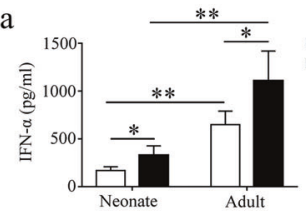

d Gated on CD45-CD31-CD326+

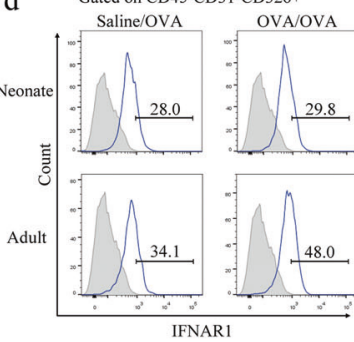

e

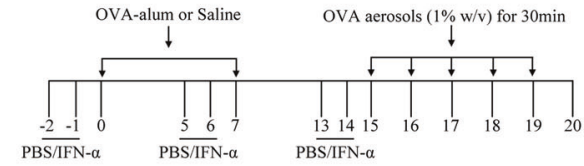

b

Isotype c
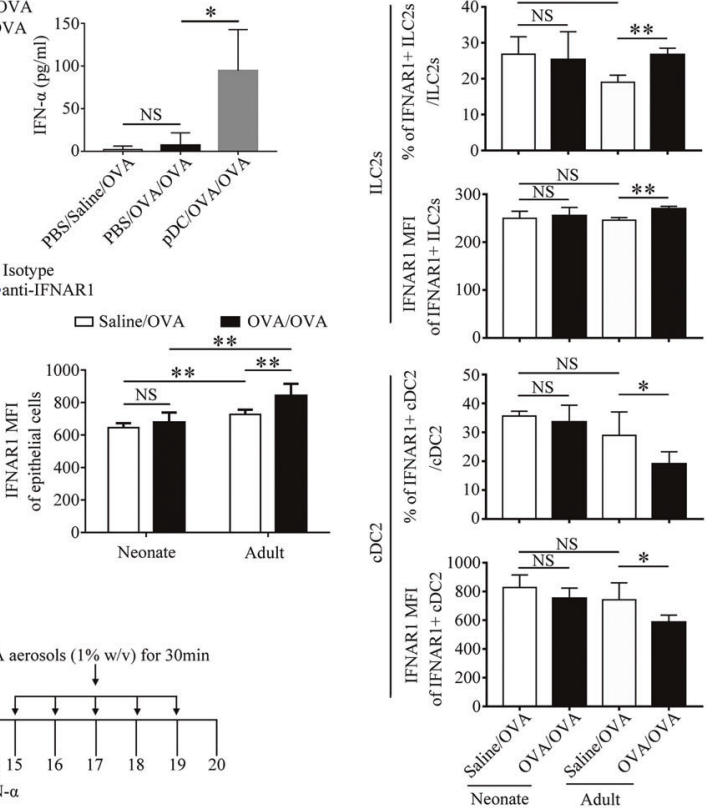

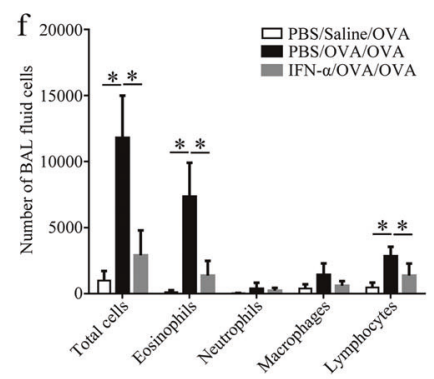

g PBS/Saline/OVA PBS/OVA/OVA IFN- $\alpha$ /OVA/OVA

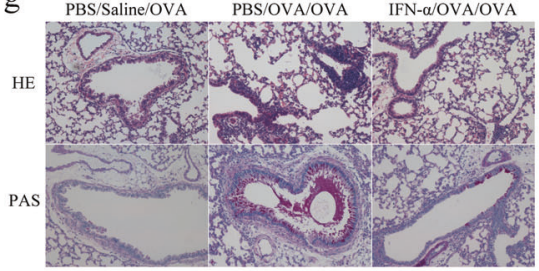

h

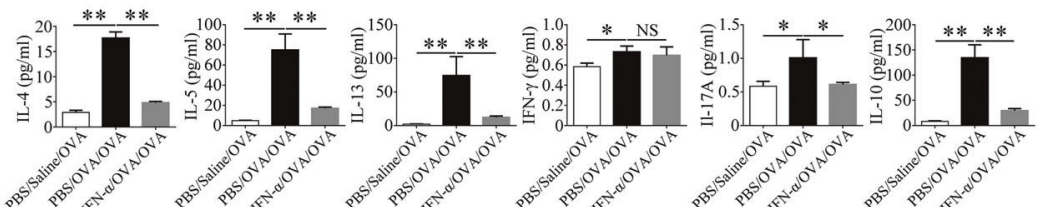

i
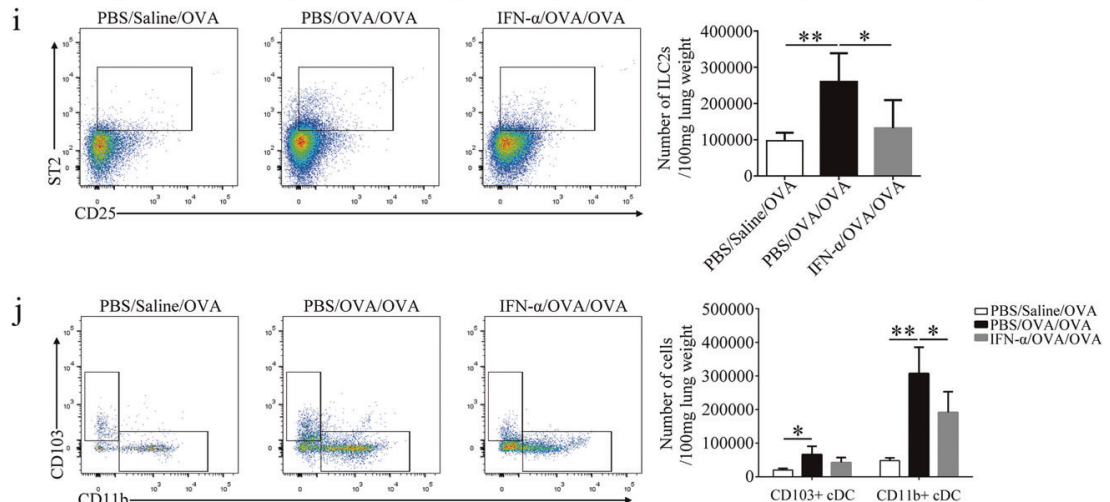

Fig. 5 Administration of IFN-a to neonatal mice reduced allergic airway responses. Neonatal and adult mice were treated as illustrated in Fig. $1 \mathrm{a}$, and $24 \mathrm{~h}$ after the final challenge, the concentration of IFN- $\alpha$ in the lung homogenates was determined by ELISA (a), the expression of IFNAR1 on lung ILC2 cells and CDC2 (CD1 $\left.1 b^{+} \mathrm{CDCs}\right)(\mathbf{c})$ and on airway epithelial cells (d) was determined by flow cytometry. $\mathbf{b}$ Neonatal mice were treated as illustrated in Fig. 3a, and $24 \mathrm{~h}$ after the final challenge, the concentration of IFN- $\alpha$ in the BAL fluid was determined by ELISA. e As shown in the time line, the neonatal mice were administered of PBS or IFN- $\alpha\left(5 \times 10^{3}\right.$ units/mouse per day, i.p. injection) twice before each sensitization and the first challenge. Mice that received PBS served as the control. The mice were dissected, and the lungs were analyzed on day 20. $\mathbf{f}$ The number of eosinophils and neutrophils in the BAL fluid was determined by flow cytometry. $\mathbf{g}$ HE- and PAS-stained lung sections. $\mathbf{h}$ Cytokine production in the supernatants of the PBLN cells 3 days after restimulation with OVA in vitro. $\mathbf{i}$, $\mathbf{j}$ The number of ILC2 cells (i) and CDCs (j) in the lung was determined by flow cytometry. All data were analyzed with Mann Whitney $U$ tests without multiple comparison correction. The results are shown as the means \pm SD $\left(n=6-7\right.$ each). ${ }^{*} P<0.05 .{ }^{* *} P<0.01$. NS not significant, MFI median fluorescence intensity. 
than it was in the adult group. The number of $\mathrm{CD}_{103^{+}} \mathrm{CDCs}$, though much lower than the $\mathrm{CD}_{11} \mathrm{~b}^{+} \mathrm{CDCs}$, was similarly increased after OVA sensitization and challenge, and there were no differences between the two age groups (Fig. 1e). Strikingly, the number of lung pDCs in the saline-sensitized neonatal mice was much lower than it was in the adult mice and did not increase upon OVA sensitization (Fig. 1g). In contrast, the number of lung pDCs in the adult mice increased significantly after OVA sensitization. As pDCs have been reported to have regulatory effects on allergen-induced airway inflammation, ${ }^{9-11}$ we further analyzed the number of pDCs in the lung and blood at a steady state in the mice of different ages. Lung pDCs were scarcely detectable in the neonates, and the number of lung pDCs increased with mouse age (Fig. 1h). The percentages of the pDCs in the peripheral blood samples showed similar trends (Supplementary Fig. 3).

Recent studies have shown that ILC2 cells, an important subset of innate immune cells in the lung, also contributes to the development of allergic airway inflammation, especially in young mice. ${ }^{27-29}$ In line with these observations, we found that the number of lung ILC2 cells increased after OVA sensitization and challenge in both age groups but was higher in the neonatal mice than in the older mice (Fig. 1f).

\section{Administration of Flt3L reduces allergic airway responses in neonatal mice}

Based on these results, we hypothesized that the relative deficiency of pDCs in neonates might contribute to the enhanced allergic airway inflammation following OVA sensitization and challenge. To test this hypothesis, we administered Flt3L two times before each sensitization injection with the goal of increasing the number of pDCs in the neonates ${ }^{30-33}$ (Fig. 2a). As expected, Flt3L treatment significantly increased the number of pDCs (Fig. 2b) but not the number of $C D 11 b^{+} \mathrm{cDCs}$ or $\mathrm{CD} 103^{+}$ CDCs (Fig. 2c) in the treated neonates. After OVA sensitization and challenge, the Flt3L-treated mice did not develop the enhanced lung allergic responses observed in the sham-treated mice. As illustrated in Fig. 2, the number of eosinophils, neutrophils, lymphocytes, and macrophages in the BAL fluid (Fig. 2d) and in the lung (Supplementary Fig. 4a), the infiltration of inflammatory cells around the airways and blood vessels, and the extent of goblet cell metaplasia (Fig. 2e) were much lower in the Flt3Ltreated mice than they were in sham-treated mice. In parallel, the levels of Th2 cytokines, IL-17 and IL-10 in the culture supernatants from the OVA-restimulated PBLN cells were also lower in the Flt3Ltreated mice than in the sham-treated mice (Fig. 2f). Notably, the Flt3L treatment also resulted in fewer ILC2 cells in the lungs (Fig. 2g). The total serum IgE and IgG1 concentrations (Supplementary Fig. $4 b, c)$ in the Flt3L-treated mice were comparable to those of the control mice.

\section{Adoptive transfer of pDCs reduces allergic airway responses in neonatal mice}

Given potential concerns that the Flt3L receptor was expressed in other cells, not only pDCs, such as hematopoietic stem cells and progenitor cells, the injection of Flt3L may have effects on cells other than $\mathrm{pDCs}$. To test whether pDCs alone were sufficient to confer protection to the neonatal mice, bone marrow-derived pDCs from wild-type mice were purified (Supplementary Fig. 5a) and adoptively transferred into the neonatal mice prior to the initial sensitization injection (Fig. 3a). The number of lung pDCs was significantly increased after the adoptive transfer of the pDCs (Fig. 3b). Neonatal mice with sham-transferred injections developed airway eosinophilia and goblet cell hyperplasia, consistent with increased production of IL-4, IL-5, and IL-13 in the cultures of the PBLN cells restimulated with OVA ex vivo. The total serum levels of IgE and IgG1 (Fig. 3c-e, Supplementary Fig. 5b-d) were also increased. All of these responses were lower in the neonatal
pDC recipients. The number of ILC2 cells and CD11 $b^{+} \mathrm{CDC}$ in the lungs of the $\mathrm{pDC}$ recipients was comparable to that in the lungs of the negative controls and much lower than that in the lungs of the mouse recipients of the sham transfer (Fig. $3 f, g$ ).

To test whether Tregs are involved in the pDC-mediated regulation mechanism, we compared the number and function of the Tregs. As illustrated in Supplementary Fig. 5e, the percentage of $\mathrm{CD}^{+}{ }^{+} \mathrm{CD} 25^{+} \mathrm{Foxp}^{+}$and IL- $10^{+} \mathrm{CD} 4^{+} \mathrm{T}$ cells was significantly increased following OVA sensitization and challenge. However, there were no differences in the levels between the pDC-recipient group and the negative control group. The percentages of the TGF- $\beta{ }^{+} \mathrm{CD}^{+}{ }^{+} \mathrm{T}$ and $\mathrm{GARP}^{+} \mathrm{CD} 4^{+} \mathrm{T}$ cells among all three groups were similar.

Selective removal of pDCs enhances allergic airway responses in adult mice

Given that the deficiency of pDCs in neonates appeared to contribute to enhanced allergic airway inflammation, we examined the effects of pDC depletion in adult mice. To this end, $120 \mathrm{G} 8$ or control lgG1 were given to adult mice during sensitization and challenge to deplete the lung pDCs (Fig. 4a). As shown in Fig. 4b, the number of pDCs was reduced after the 120G8 treatment. The IgG1-treated OVA-sensitized control mice developed typical Th2 cell-associated airway inflammation (Fig. 4c-e, Supplementary Fig. 6). The 120G8-treated mice had enhanced increases in airway eosinophilia in the lungs and BAL fluid, as well as increases in the peri-bronchovascular infiltrates and goblet cell metaplasia. These changes were accompanied by higher levels of Th2 cytokines and IL-10 in the OVA-restimulated PBLN culture supernatants (Fig. 4c-e, Supplementary Fig. 6a). Thus, the selective removal of the pDCs from adult mice enhanced the Th2 cell-associated allergic airway inflammatory responses.

To determine if these changes were associated with alterations in the number of innate immune cells, the number of lung ILC2 cells and $C D 11 b^{+}$cDCs was determined. We found that the number of both cell types was significantly higher after OVA sensitization and challenge and was further increased in the 120G8-treated mice (Fig. 4f, g).

Administration of IFN-a reduces allergic airway responses in neonatal mice

pDCs are a major source of IFN-a. A recent study reported that pDC may have a regulatory role in allergic airway responses by secreting IFN-a. ${ }^{11}$ By monitoring the levels of IFN-a in the lungs and BAL fluid as well as the expression of IFNAR1 on lung inflammatory cells and epithelial cells in the neonatal and adult mice after OVA sensitization and challenge, we found that in the nonsensitized but challenged groups, the levels of lung IFN- $a$ in the adults were higher than those in the neonates. OVA sensitization and challenge induced further increases in the levels of IFN-a in both age groups, and the IFN-a levels were higher in the adults than in the neonates (Fig. 5a). In the neonatal mice, the levels of IFN- $a$ in the BAL fluid were similar between the OVA-sensitized and sham-sensitized groups. The transfer of adult pDCs to the neonatal mice before OVA sensitization significantly increased the concentration of IFN- $\alpha$ in the BAL fluid (Fig. 5b).

In the neonates, the expression of IFNAR1 on ILC2 cells, CD11 b ${ }^{+}$ CDCs (cDC2 cells), and epithelial cells was not different between the saline-treated and the OVA-sensitized mice. However, in the OVA-challenged adult mice, the expression of IFNAR1 was higher on the ILC2 and epithelial cells but lower on the CD11 b $b^{+}$CDCs compared to the levels in the controls (Fig. 5c, d). When the two age groups were compared, the expression levels of IFNAR1 on the lung epithelial cells were higher in the adult groups than in the neonatal groups (Fig. 5d), whereas the percentages of IFNAR1expressing ILC2 cells were higher in the saline-sensitized neonatal group (Fig. 5c). 


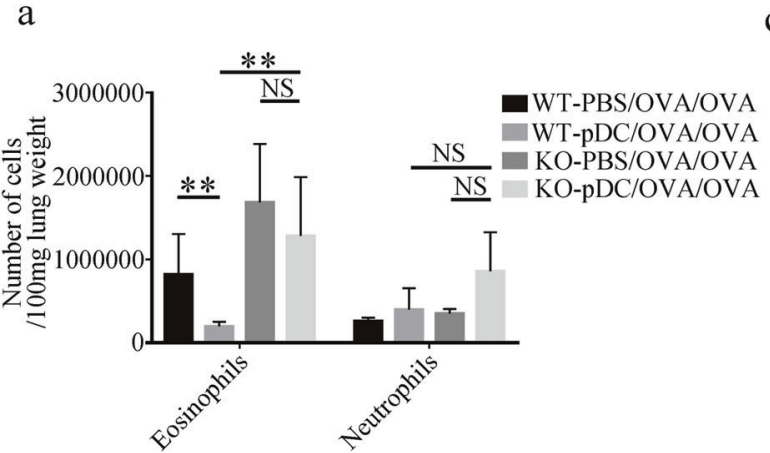

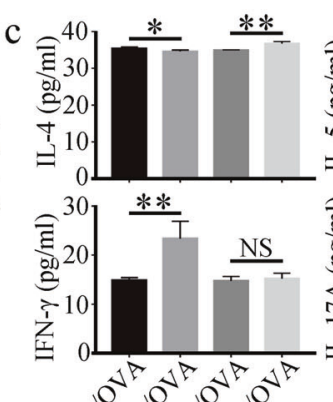

ats at at a
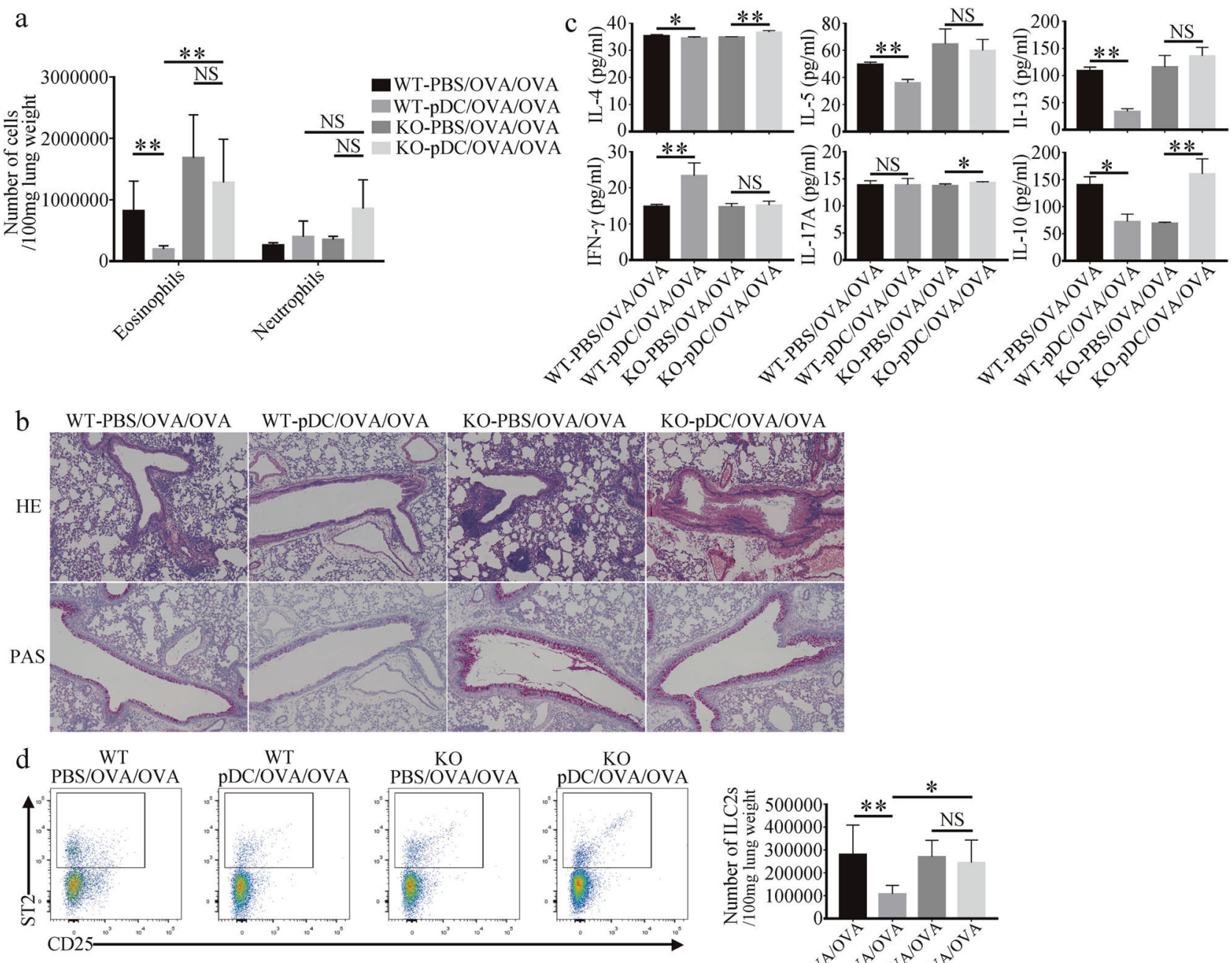

ation 0

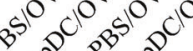

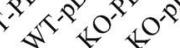
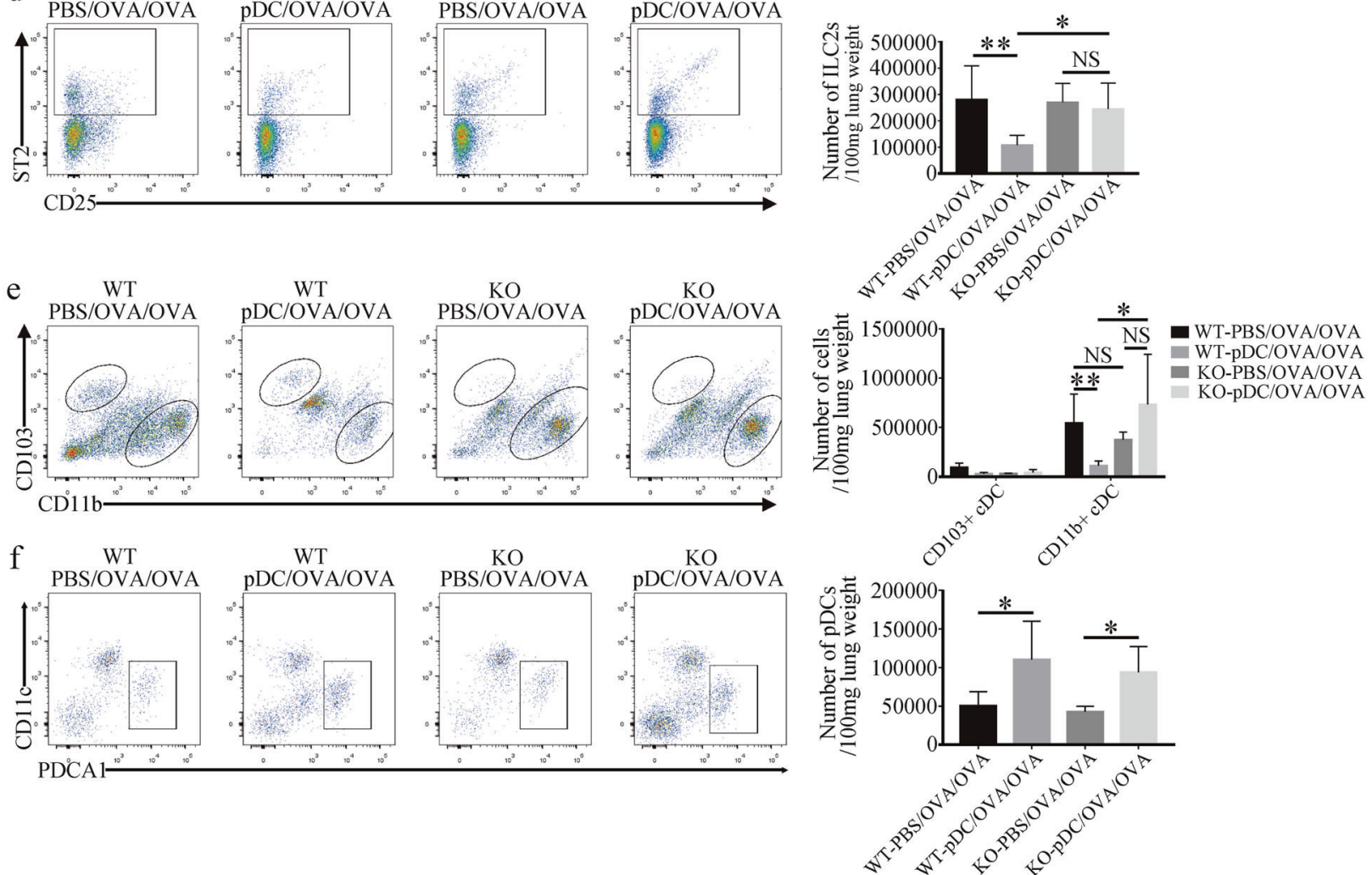

Fig. 6 Adoptive transfer of pDCs to IFNAR1-knockout neonatal mice failed to reduce allergic airway responses. Sorted pDCs were transferred to wild type and IFNAR $1^{-1-}$ neonatal mice before OVA sensitization, as illustrated in Fig. 3a. The mice were dissected, and the lungs were analyzed on day 20. a The number of eosinophils and neutrophils in the lung was determined by flow cytometry. $\mathbf{b}$ HE- and PASstained lung sections. $\mathbf{c}$ Cytokine production in the supernatants of the PBLN cells 3 days after restimulation with OVA in vitro. $\mathbf{d}-\mathbf{f}$ The number of ILC2 cells (d), CDCs (e), and pDCs (f) in the lungs was determined by flow cytometry. All data were analyzed with Mann Whitney $U$ tests without multiple comparison correction. The results are shown as the means \pm SD $\left(n=5-8\right.$ each). ${ }^{*} P<0.05$. ${ }^{* *} P<0.01$. NS not significant, WT wild type, KO IFNAR1 knockout. 

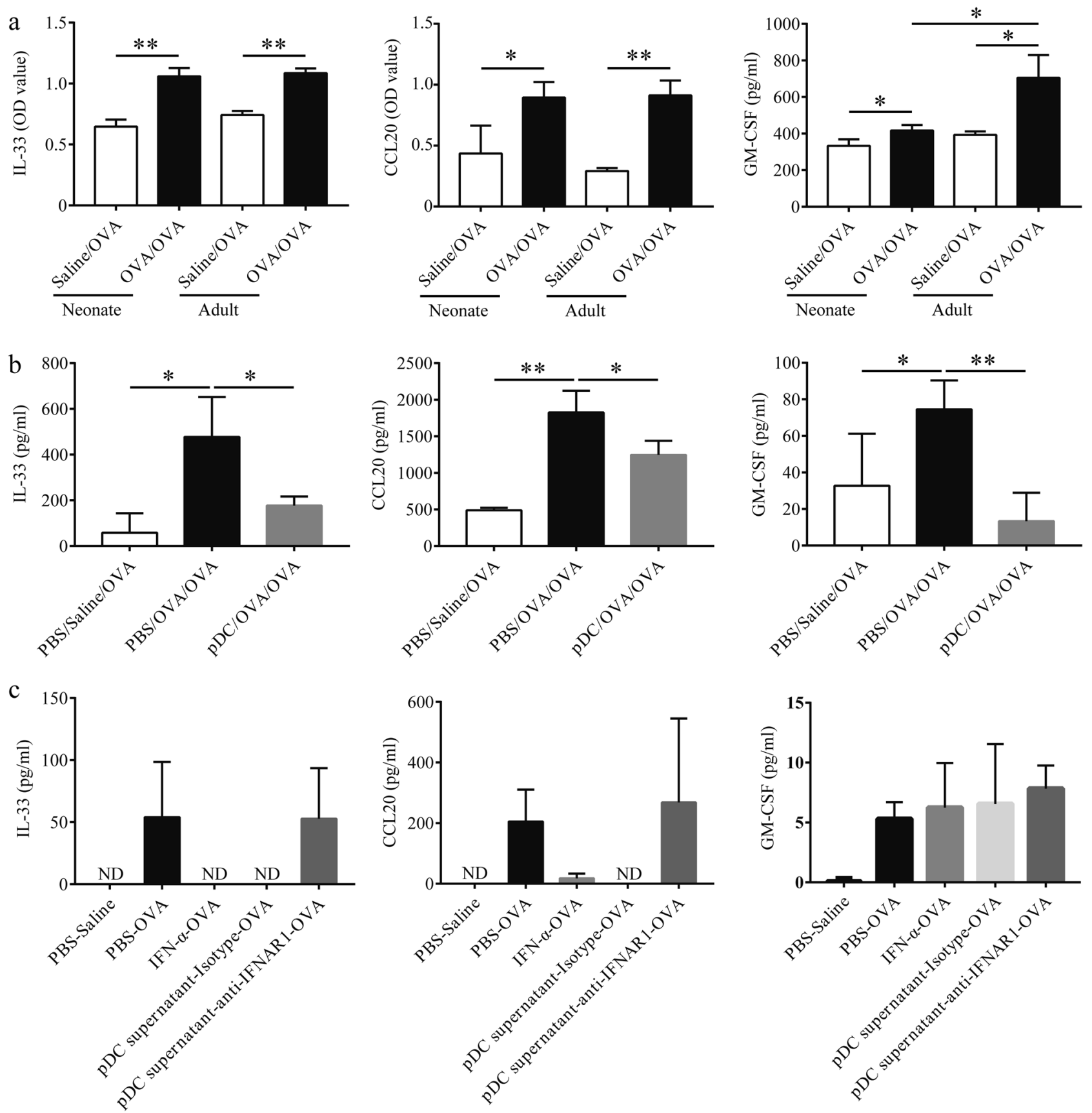

Fig. 7 pDCs suppressed the secretion of IL-33, CCL20 and GM-CSF by airway epithelial cells. a Neonatal and adult mice were treated as illustrated in Fig. $1 \mathrm{a}$, and $24 \mathrm{~h}$ after the final challenge, the concentrations of IL-33, CCL20, and GM-CSF in the lung homogenates were determined by ELISA. b Neonatal mice were treated as illustrated in Fig. 3a, and $24 \mathrm{~h}$ after the final challenge, the concentrations of IL-33, CCL20, and GM-CSF in the BAL fluid were determined by ELISA. c Epithelial cells were sorted and cocultured with OVA in the presence of IFN$\alpha, \mathrm{pDC}$ culture supernatant, or pDC culture supernatant plus anti-IFNAR1 mAb for $72 \mathrm{~h}$. The supernatants were collected, and the concentrations of IL-33, CCL20, and GM-CSF were determined by ELISA. All data were analyzed with Mann Whitney $U$ tests without multiple comparison correction. The results are shown as the means \pm SD. ${ }^{*} P<0.05$. ${ }^{* *} P<0.01$. ND not detected.

We administered IFN- $a$ to neonatal mice during the sensitization and challenge phase (Fig. 5e). Similar to the pDC adoptive transfer experiment, IFN-a administration significantly inhibited the development of Th2 cell-associated airway inflammation (Fig. 5f-h, Supplementary Fig. 7). When lung ILC2 cells and DC subsets were examined, the number of ILC2 cells, CD103 ${ }^{+} \mathrm{CDCs}$, and $C D 11 b^{+} \mathrm{cDCs}$ in the lungs of the OVA-sensitized neonatal mice was higher than it was in the saline-sensitized mice (Fig. $5 i$, j). IFN-a treatment significantly reduced the number of ILC2 cells and CD $11 b^{+}$cDCs.
The protective role of pDCs is dependent on the expression of IFNAR1

To determine whether pDCs exert their function through IFNAR1, isolated $\mathrm{pDC}$ s were transferred into wild - type or IFNAR1 ${ }^{-1-}$ neonatal mice before OVA sensitization (Fig. 3a). After the final challenge, the wild-type recipients developed markedly reduced allergic inflammation, but this protective effect was not observed in the IFNAR1 ${ }^{-1-}$ recipients (Fig. 6a-c, Supplementary Fig. 8). Consistently, lower number of ILC2 cells and CD11 b ${ }^{+}$CDCs in the lung was detected in wild-type recipients, whereas this number 
530
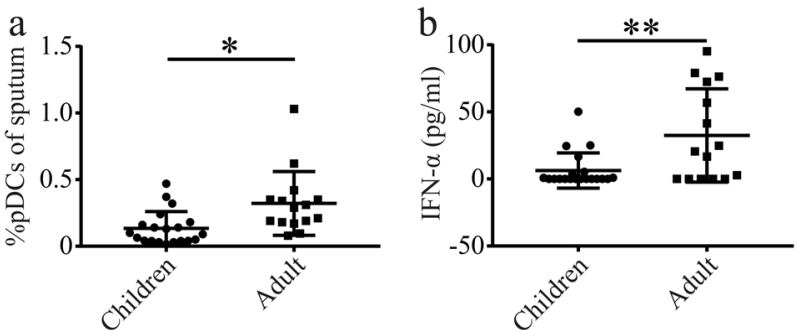

Fig. 8 Sputum pDC numbers were lower in children with asthma and were associated with lower levels of IFN-a. a The percentage of pDCs in the induced sputum collected from children and adult patients with asthma was determined by flow cytometry. b The concentration of IFN- $\alpha$ in the induced sputum was determined by ELISA. All data were analyzed with Mann Whitney $U$ tests without multiple comparison correction. The results are shown as the means \pm SD (children, $n=22$; adults, $n=15$ ). ${ }^{*} P<0.05$.

was much higher in the IFNAR1 ${ }^{-1-}$ recipients (Fig. $6 \mathrm{~d}$, e). The survival and homeostasis of the pDCs were not affected, as the number of pDCs in the lung was comparable between the wild type and IFNAR1 ${ }^{-1-}$ recipient mice, and the number in both groups was higher than that in the sham-transfer control group (Fig. 6f).

pDCs regulate allergic responses by suppressing the secretion of inflammatory cytokines from epithelial cells

The data suggested that the deficiency of pDCs in neonates contributed to enhanced allergic airway inflammation and was associated with a decreased number of ILC2 cells and CD11 $\mathrm{b}^{+}$ $\mathrm{CDCs}$ in the lungs. Previous studies demonstrated that IL-33, CCL20, and GM-CSF, when released by epithelial cells upon allergen stimulation, act on $\mathrm{CDCs}$ and promote Th2 cell differentiation. In addition, epithelial cell-derived IL-33 stimulated ILC2 cells. $^{34,35}$ Based on these findings, we determined whether epithelial cell-derived cytokines were involved in the protective effects of pDCs on airway inflammation. To make this determination, we examined the levels of epithelial cell-derived cytokines in lung homogenates. In both age groups, the levels of lung IL-33, CCL20 and GM-CSF increased following OVA sensitization and challenge compared with levels in the saline-sensitized controls (Fig. 7a). Adoptive transfer of pDCs resulted in lower levels of these cytokines in the BAL fluid from the neonatal mice (Fig. 7b). The concentrations of lung GM-CSF in the adult mice were higher than those in the neonates after OVA sensitization and challenge. These results implicated pDCs in the regulation of the production of epithelial cell-derived cytokines, which could in turn reduce airway inflammation.

To directly define the interaction between pDCs and epithelial cells, the sorted epithelial cells were stimulated with OVA or saline for $72 \mathrm{~h}$. As shown in Fig. 7c, the concentrations of IL-33 and CCL20 in the OVA-stimulated cell supernatants were significantly higher than they were in the saline-stimulated cell supernatants. When IFN-a or pDC culture supernatants were added to the culture, the levels of IL-33 and CCL20 were markedly reduced. This suppressive effect was completely lost after the addition of antiIFNAR1. The concentrations of GM-CSF were not altered. Taken together, these results suggest that $\mathrm{pDCs}$ play a regulatory role through the suppression of inflammatory cytokine production from epithelial cells.

Sputum pDC numbers are lower in children with asthma and are associated with lower levels of IFN-a

To determine whether the role of pDCs established in the mouse model is conserved in asthma patients, we analyzed the number of pDCs and the levels of IFN- $a$ in the induced sputum samples obtained from asthma patients of different ages (Tables S1 and
S2). In both age groups, asthma was associated with airway eosinophilia, ${ }^{36}$ as the percentage of eosinophils was $>3 \%$, while the percentage of neutrophils was $<76 \%$ (Supplementary Fig. 9a, b). As shown in Fig. 8a, b and Supplementary Fig. 9c, the percentages of pDCs were lower in children with asthma than they were in the adult patients, and these levels were associated with lower levels of IFN-a. This finding is similar to that observed in the sensitized and challenged neonatal mice.

\section{DISCUSSION}

Allergic asthma often begins in early life, and in mouse studies, the earlier the initial event, the more significant the responses are to subsequent exposure. ${ }^{37}$ In the present study, mice were sensitized as neonates to mimic, as best as possible, early-onset childhood allergic asthma. We found that allergen sensitization in neonatal mice differed from that in adult mice in that the neonatal mice had a more pronounced allergic airway response after allergen challenge. In parallel, the sensitized neonatal mice had higher levels of IL-4, IL-5 and IL-13, serum IgE levels and lower levels of IFN- $\gamma$ production. These data suggest that early sensitization results in more pronounced Th2 cell responses, confirming and extending previous studies. ${ }^{34,37}$ Many factors could contribute to these Th2 cell-dominant responses at an early age. In neonatal mice, heightened lung allergic responses to allergens may be due to higher concentrations of IL-33 in the lungs. 34

Based on earlier findings, ${ }^{17}$ we focused on a subset of innate immune cells, pDCs, in this study. We found that there were very few pDCs in the lungs and blood of neonatal mice, and the percentages of pDCs in the lung and blood increased with age. pDCs have been shown to have a negative regulatory function in airway allergic inflammation in adult mice. ${ }^{11,23}$ However, a thorough understanding of the regulatory role of pDCs, especially in neonates, is still lacking. We speculated that the enhanced allergic airway responses shown by neonatal mice resulted from deficient pDCs and the lowered function as a consequence. To test this hypothesis, we replenished neonatal mice with pDCs either by Flt3L treatment to induce generation of endogenous pDCs or by adoptive transfer of exogenous pDCs from adult mice. Both approaches were found to significantly reduce the development of allergic airway responses, indicating that supplementation of pDCs before allergen sensitization was sufficient to abolish the development of allergen-induced allergic airway responses. Whether cell activation is needed for pDCs to exert their regulatory function is not clear. In the present study, the pDCs were not activated before adoptive transfer, a finding that is consistent with those of previous reports. ${ }^{7}$ Moreover, in corollary experiments, we demonstrated that depletion of pDCs in adult mice during allergen sensitization and challenge significantly enhanced allergic airway responses. Together, these findings led to the identification of a critical role for pDCs in regulating the development of allergic asthma.

Previous studies have shown that pDCs play a regulatory role in allergic asthma through multiple mechanisms, including the induction of Tregs, ${ }^{7,9}$ activation of the PD1/PD-L1 pathway, ${ }^{23}$ and secretion of IFN-a. ${ }^{11}$ Here, we showed that pDCs exert their suppressive function via the secretion of IFN- $a$, as IFN- $a$ administration recapitulated the suppressive effects of transferred pDCs on the development of allergic airway inflammation. In support of this hypothesis, we also found that the concentrations of IFN- $a$ in the lungs of the adult mice were higher than they were in the neonatal mice. Furthermore, pDC transfer before allergen sensitization resulted in increased concentrations of IFN- $a$ in the BAL fluid from neonatal mice and increased protective effects, whereas transfer of pDCs to IFNAR1 ${ }^{-1-}$ mice did not show any benefit. Notably, it has been shown that cross-linking lgE on the surface of human pDCs impairs their capacity to secrete IFN-a. ${ }^{38-41}$ This represents a 
potential mechanism by which allergic asthma patients are more susceptible to asthma exacerbation. In our study, the pDCs were modified before OVA challenge. At this time point, the allergic responses are not fully developed, and the levels of $\operatorname{lgE}$ are low. Hence, the effect of $\operatorname{lgE}$ on $\mathrm{pDC}$ function would be minimal. However, the higher levels of IgE following OVA challenge in the sensitized neonatal group may have impaired IFN-a production from the pDCs, which would render children with asthma more vulnerable to virus infection and asthma exacerbation. In addition to the effects of IFN- $a$, we also determined the involvement of Tregs. We found that OVA sensitization and challenge induced an increase in $\mathrm{CD}^{+}{ }^{+} \mathrm{CD} 25^{+} \mathrm{Foxp}^{+}$Treg and IL-10 production. However, no differences were found between the pDC-recipient group and the negative control group. Since the role of Tregs was studied at $24 \mathrm{~h}$ after the last challenge, a time point at which their influence may be limited, we cannot rule out the possibility that pDC-Treg interactions are involved earlier than could be captured in the experimental scheme.

An inverse correlation in the number of ILC2 cells and the number of pDCs was evident in the neonatal mice, which had fewer pDCs and more ILC2 cells in the lungs compared to the levels in the adult mice after OVA sensitization and challenge. Adoptive transfer of the pDCs or administration of IFN- $a$ to these sensitized mice resulted in a reduced number of ILC2 cells after the OVA challenge. In contrast, the adult mice had more pDCs and fewer ILC2 cells compared to the number of each in the neonatal mice. pDC depletion in sensitized adult mice resulted in increases in ILC2 cells after the OVA challenge. These results suggest a possible counteracting regulation between these two cell types. ILC2 cells express IFNAR1, and there is evidence showing that type I and II interferons have a regulatory role on ILC2 cells. ${ }^{42-44}$ pDCs, through IFN-a, can suppress cytokine production and increase the apoptosis rate of ILC2 cells. ${ }^{11}$ Since ILC2 cells have been identified as significant producers of type 2 cytokines and contributors to allergic asthma, ${ }^{45,46}$ it is perhaps not surprising that the reduction in ILC2 cells was accompanied by less severe airway allergic inflammation. Thus, one possibility is that more ILC2 cells in neonates may be associated with deficiency in pDCs and IFN-a, thus contributing to the development of asthma.

The importance of DCs in antigen presentation, Th2 cell polarization, and recruitment of effector cells to the lung has been well described. ${ }^{47,48}$ Several subsets of DCs reside in the lung. The number of $\mathrm{CD}_{103^{+}} \mathrm{CDCs}$, one DC subset, did not substantially change in the neonates or adult mice after sensitization. CD11 b cDCs represent the major subset to initiate and maintain Th2 cell responses. Interestingly, although OVA-induced allergic responses were more severe in the neonatal group, the number of $C D 11 b^{+}$ CDCs was lower than in the adult group. A similar phenomenon has been described in a recent study using an HDM model ${ }^{34}$ in which neonatal $\mathrm{CD} 11 \mathrm{~b}^{+} \mathrm{cDCs}$ were shown to have a stronger "proTh2" cell activity than observed in the adult $C D 11 b^{+} c D C s$. These findings further confirmed that the function of $C D 11 b^{+} \mathrm{cDCs}$ is important in airway allergic responses. In the current study, we found that lung $\mathrm{CD} 11 \mathrm{~b}^{+} \mathrm{CDCs}$ followed the same trend as the ILC2 cells. Similarly, ILC2 cells have been shown to license dendritic cells to potentiate Th2 memory cell responses; ${ }^{49}$ it is possible that the higher number of ILC2 cells in the neonates augmented the CDC activity to initiate more pronounced Th2 cell responses.

An airway epithelial cell/ILC2 cell/DC axis has been suggested to play an important role in the development of allergic airway inflammation, but the role of pDCs has not been fully appreciated. We defined several lines of evidence indicating an important interplay between pDCs and lung epithelial cells. First, epithelial cells express IFNAR1. Second, the production of IL-33, CCL20, and GM-CSF in the lungs of the neonatal and adult mice was increased after allergen sensitization and challenge, and adoptive transfer of pDCs significantly reduced the levels of these epithelial cell-secreted cytokines. Finally, the results from the in vitro experiments showed that allergen stimulation increased the secretion of IL-33 and CCL20 from epithelial cells, while addition of IFN- $a$ or pDCs culture supernatants reduced the concentrations of these two cytokines; this reduction was attenuated when anti-IFNAR1 was included in the culture system. These findings suggested that pDCs regulate the function of airway epithelial cells through IFN-a-IFNAR1 interactions. A mechanism by which IFN-a-mitigated Th2 cell-biased responses in neonates has been suggested in an RSV reinfection model. ${ }^{18}$ IFN-a treatment prior to RSV infection downregulated the expression of IL$4 \mathrm{Ra}$ on Th2 cells and substantially reduced airway responses upon reinfection. In the current study, we propose that pDCs exert a negative regulatory role with IFN-a acting on epithelial cells to suppress the secretion of IL-33, CCL20, and GM-CSF, known activators of ILC2 cells and CD11 b ${ }^{+} \mathrm{CDCs}$, respectively. ${ }^{50,51}$

It is often difficult to extrapolate findings in experimental models to human asthma. As a proof of principle, we measured sputum pDCs, eosinophils, and IFN-a levels in children with asthma and compared the findings to samples from adults with asthma. The percentages of pDCs and the levels of IFN-a were lower in the children than in adult patients, findings that are similar to those established in the sensitized and challenged neonatal mice. However, this study has some limitations. First, we did not address pDC functions. Future studies to compare pDC functions in these two age groups, such as the capability to produce IFN-a upon stimulation or in coculture with naïve CD4 ${ }^{+}$ $\mathrm{T}$ cells, are required to elucidate the possible regulatory mechanisms. Second, as we noted, other factors could be involved, for example, the level of lgE might also be related to the observations made in this study. Finally, the severity of the asthma was not indicated in the subject enrollment criteria, limiting our ability to determine the correlation between pDCs and asthma severity. Future studies will require larger sample sizes to give it sufficient power for subgroup analyses.

In conclusion, our results suggest that $\mathrm{pDCs}$ negatively regulate allergic airway inflammation and type 2 immune responses in an IFN-a-/IFNAR1-dependent manner. Because of the deficiency of $\mathrm{pDCs}$, neonatal mice demonstrate unrestrained production of IL33, CCL20, and GM-CSF by airway epithelial cells, which together with increased numbers of ILC2 cells and CD11 $b^{+} \mathrm{CDC}$ s resulted in enhanced Th2 immune responses. This characteristic renders neonatal mice prone to develop more severe allergic airway responses upon allergen exposure. In light of the similar reduction in the number of pDCs and the IFN-a levels in children with asthma, this study provides a foundation for a better understanding of the mechanisms whereby infants exhibit increased susceptibility to the development of allergic immune responses in the lungs.

\section{ACKNOWLEDGEMENTS}

We thank Dr. Bo Zhong (University of Wuhan, Wuhan, China) for sharing the IFNAR1 ${ }^{-1-}$ mice. This work was supported by grants 91542103 and 31770994 from the National Natural Science Foundation of China (to J.H.) and grant 2015CFB620 from the Natural Science Foundation of Hubei Province (to J.H.).

\section{AUTHOR CONTRIBUTIONS}

M.W., Y.H., and J.H. conceptualized the study. M.W., L.G., M.H., H.L., H.J., K.S., and R.S. carried out the investigations. M.W., E.W.G., Y.H., and J.H. conducted formal analyses of the data. B.L., S.G., and H.C. provided resources. J.H. acquired funding and supervised the study. M.W., B.L., S.G., H.C., F.G., E.W.G., Y.H., and J.H. prepared the figures and wrote the manuscript.

\section{ADDITIONAL INFORMATION}

The online version of this article (https://doi.org/10.1038/s41423-019-0333-y) contains supplementary material.

Competing interests: The authors declare no competing interests. 


\section{REFERENCES}

1. Mjosberg, J. M. et al. Human IL-25- and IL-33-responsive type 2 innate lymphoid cells are defined by expression of CRTH2 and CD161. Nat. Immunol. 12, 1055-1062 (2011).

2. Neill, D. R. et al. Nuocytes represent a new innate effector leukocyte that mediates type-2 immunity. Nature 464, 1367-1370 (2010).

3. Gervassi, A. L. \& Horton, H. Is infant immunity actively suppressed or immature? Virology 2014, 1-9 (2014).

4. Zaghouani, H., Hoeman, C. M. \& Adkins, B. Neonatal immunity: faulty T-helpers and the shortcomings of dendritic cells. Trends Immunol. 30, 585-591 (2009).

5. Gilliet, M., Cao, W. \& Liu, Y. J. Plasmacytoid dendritic cells: sensing nucleic acids in viral infection and autoimmune diseases. Nat. Rev. Immunol. 8, 594-606 (2008).

6. Guiducci, C. et al. TLR recognition of self nucleic acids hampers glucocorticoid activity in lupus. Nature 465, 937-941 (2010).

7. de Heer, H. J. et al. Essential role of lung plasmacytoid dendritic cells in preventing asthmatic reactions to harmless inhaled antigen. J. Exp. Med. 200, 89-98 (2004).

8. Hadeiba, $\mathrm{H}$. et al. Plasmacytoid dendritic cells transport peripheral antigens to the thymus to promote central tolerance. Immunity 36, 438-450 (2012).

9. Lombardi, V., Speak, A. O., Kerzerho, J., Szely, N. \& Akbari, O. CD8alpha(+)beta(-) and CD8alpha(+)beta(+) plasmacytoid dendritic cells induce Foxp3(+) regulatory $\mathrm{T}$ cells and prevent the induction of airway hyper-reactivity. Mucosal Immunol. 5, 432-443 (2012).

10. Ouabed, A. et al. Differential control of $T$ regulatory cell proliferation and suppressive activity by mature plasmacytoid versus conventional spleen dendritic cells. J. Immunol. 180, 5862-5870 (2008).

11. Maazi, H. et al. Activated plasmacytoid dendritic cells regulate type 2 innate lymphoid cell-mediated airway hyperreactivity. J. Allergy Clin. Immunol. 141, 893-905.e896 (2018)

12. Danis, B. et al. Interferon regulatory factor 7-mediated responses are defective in cord blood plasmacytoid dendritic cells. Eur. J. Immunol. 38, 507-517 (2008).

13. De Wit, D. et al. Blood plasmacytoid dendritic cell responses to CpG oligodeoxynucleotides are impaired in human newborns. Blood 103, 1030-1032 (2004).

14. Gold, M. C., Donnelly, E., Cook, M. S., Leclair, C. M. \& Lewinsohn, D. A. Purified neonatal plasmacytoid dendritic cells overcome intrinsic maturation defect with TLR agonist stimulation. Pediatr. Res. 60, 34-37 (2006).

15. Kollmann, T. R. et al. Neonatal innate TLR-mediated responses are distinct from those of adults. J. Immunol. 183, 7150-7160 (2009).

16. Zhang, $X$. et al. Neonatal plasmacytoid dendritic cells (pDCs) display subset variation but can elicit potent anti-viral innate responses. PLOS ONE 8, e52003 (2013).

17. Han, J. et al. Responsiveness to respiratory syncytial virus in neonates is mediated through thymic stromal lymphopoietin and OX40 ligand. J. Allergy Clin. Immunol. 130, 1175-1186.e1179 (2012).

18. Cormier, S. A. et al. Limited type I interferons and plasmacytoid dendritic cells during neonatal respiratory syncytial virus infection permit immunopathogenesis upon reinfection. J. Virol. 88, 9350-9360 (2014).

19. Debeuf, N., Haspeslagh, E., van Helden, M., Hammad, H. \& Lambrecht, B. N. Mouse models of asthma. Curr. Protoc. Mouse Biol. 6, 169-184 (2016).

20. Semitekolou, M. et al. Activin-A induces regulatory $T$ cells that suppress $T$ helper cell immune responses and protect from allergic airway disease. J. Exp. Med. 206, 1769-1785 (2009).

21. Schuijs, M. J. et al. Farm dust and endotoxin protect against allergy through A20 induction in lung epithelial cells. Science 349, 1106-1110 (2015).

22. Sinha, M. \& Lowell, C. A. Isolation of highly pure primary mouse alveolar epithelial type ii cells by flow cytometric cell sorting. Bio-protocol 6, pii:e2013 (2016).

23. Kool, M. et al. An anti-inflammatory role for plasmacytoid dendritic cells in allergic airway inflammation. J. Immunol. 183, 1074-1082 (2009).

24. Zhang, Z. et al. Plasmacytoid dendritic cells promote HIV-1-induced group 3 innate lymphoid cell depletion. J. Clin. Invest. 125, 3692-3703 (2015).

25. Dzionek, A. et al. BDCA-2, BDCA-3, and BDCA-4: three markers for distinct subsets of dendritic cells in human peripheral blood. J. Immunol. 165, 6037-6046 (2000).

26. Lee, J. J. \& McGarry, M. P. When is a mouse basophil not a basophil? Blood 109, 859-861 (2007).
27. Dhariwal, J. et al. Mucosal type 2 innate lymphoid cells are a key component of the allergic response to aeroallergens. Am. J. Respir. Crit. Care Med. 195, 1586-1596 (2017).

28. Maazi, H. et al. ICOS:ICOS-ligand interaction is required for type 2 innate lymphoid cell function, homeostasis, and induction of airway hyperreactivity. Immunity 42, 538-551 (2015).

29. Wallrapp, A. et al. The neuropeptide NMU amplifies ILC2-driven allergic lung inflammation. Nature 549, 351-356 (2017).

30. Bjorck, P. Isolation and characterization of plasmacytoid dendritic cells from Flt3 ligand and granulocyte-macrophage colony-stimulating factor-treated mice. Blood 98, 3520-3526 (2001)

31. Edwan, J. H., Perry, G., Talmadge, J. E. \& Agrawal, D. K. Flt-3 ligand reverses late allergic response and airway hyper-responsiveness in a mouse model of allergic inflammation. J. Immunol. 172, 5016-5023 (2004).

32. Edwan, J. H., Talmadge, J. E. \& Agrawal, D. K. Treatment with Flt3 ligand plasmid reverses allergic airway inflammation in ovalbumin-sensitized and -challenged mice. Int. Immunopharmacol. 5, 345-357 (2005).

33. Liu, Y. J. IPC: professional type 1 interferon-producing cells and plasmacytoid dendritic cell precursors. Annu. Rev. Immunol. 23, 275-306 (2005).

34. de Kleer, I. M. et al. Perinatal activation of the interleukin-33 pathway promotes type 2 immunity in the developing lung. Immunity 45, 1285-1298 (2016).

35. Jackson, D. J. et al. IL-33-dependent type 2 inflammation during rhinovirusinduced asthma exacerbations in vivo. Am. J. Respir. Crit. Care Med. 190, 1373-1382 (2014).

36. Schleich, F. N. et al. Distribution of sputum cellular phenotype in a large asthma cohort: predicting factors for eosinophilic vs neutrophilic inflammation. BMC Pulm. Med. 13, 11 (2013).

37. Gelfand, E. W. et al. Induction and maintenance of airway responsiveness to allergen challenge are determined at the age of initial sensitization. J. Immunol. 173, 1298-1306 (2004).

38. Durrani, S. R. et al. Innate immune responses to rhinovirus are reduced by the high-affinity IgE receptor in allergic asthmatic children. J. Allergy Clin. Immunol. 130, 489-495 (2012).

39. Gill, M. A. et al. Counterregulation between the FcepsilonRI pathway and antiviral responses in human plasmacytoid dendritic cells. J. Immunol. 184, 5999-6006 (2010).

40. Gill, M. A. et al. Enhanced plasmacytoid dendritic cell antiviral responses after omalizumab. J. Allergy Clin. Immunol. 141, 1735-1743.e1739 (2018).

41. Schroeder, J. T. et al. TLR9- and FcepsilonRI-mediated responses oppose one another in plasmacytoid dendritic cells by down-regulating receptor expression. J. Immunol. 175, 5724-5731 (2005).

42. Duerr, C. U. et al. Type I interferon restricts type 2 immunopathology through the regulation of group 2 innate lymphoid cells. Nat. Immunol. 17, 65-75 (2016).

43. Molofsky, A. B. et al. Interleukin-33 and interferon-gamma counter-regulate group 2 innate lymphoid cell activation during immune perturbation. Immunity 43, 161-174 (2015)

44. Moro, K. et al. Interferon and IL-27 antagonize the function of group 2 innate lymphoid cells and type 2 innate immune responses. Nat. Immunol. 17, 76-86 (2016).

45. Barlow, J. L. et al. Innate IL-13-producing nuocytes arise during allergic lung inflammation and contribute to airways hyperreactivity. J. Allergy Clin. Immunol. 129, 191-198 (2012). e191-194.

46. Moro, $\mathrm{K}$. et al. Innate production of $\mathrm{T}(\mathrm{H}) 2$ cytokines by adipose tissue-associated c-Kit(+)Sca-1(+) lymphoid cells. Nature 463, 540-544 (2010).

47. Plantinga, $\mathrm{M}$. et al. Conventional and monocyte-derived $\mathrm{CD} 11 \mathrm{~b}(+)$ dendritic cells initiate and maintain $\mathrm{T}$ helper 2 cell-mediated immunity to house dust mite allergen. Immunity 38, 322-335 (2013).

48. Williams, J. W. et al. Transcription factor IRF4 drives dendritic cells to promote Th2 differentiation. Nat. Commun. 4, 2990 (2013).

49. Halim, T. Y. et al. Group 2 innate lymphoid cells license dendritic cells to potentiate memory TH2 cell responses. Nat. Immunol. 17, 57-64 (2016).

50. Lambrecht, B. N. \& Hammad, H. The immunology of asthma. Nat. Immunol. 16, 45-56 (2015).

51. Worbs, T., Hammerschmidt, S. I. \& Forster, R. Dendritic cell migration in health and disease. Nat. Rev. Immunol. 17, 30-48 (2017). 